\title{
Two-photon absorption spectroscopy of trans-stilbene, cis-stilbene, and phenanthrene: Theory and experiment
}

\author{
Marc de Wergifosse, ${ }^{1}$ Amanda L. Houk, ${ }^{2, a)}$ Anna I. Krylov, ${ }^{1, b)}$ and Christopher G. Elles ${ }^{2, b)}$ \\ ${ }^{1}$ Department of Chemistry, University of Southern California, Los Angeles, California 90089-0482, USA \\ ${ }^{2}$ Department of Chemistry, University of Kansas, Lawrence, Kansas 66045, USA
}

(Received 13 December 2016; accepted 21 March 2017; published online 11 April 2017)

\begin{abstract}
Two-photon absorption (2PA) spectroscopy provides complementary, and sometimes more detailed, information about the electronic structure of a molecule relative to one-photon absorption (1PA) spectroscopy. However, our understanding of the 2PA processes is rather limited due to technical difficulties in measuring experimental 2PA spectra and theoretical challenges in computing higherorder molecular properties. This paper examines the 2PA spectroscopy of trans-stilbene, cis-stilbene, and phenanthrene by a combined experimental and theoretical approach. The broadband 2PA spectra of all three compounds are measured under identical conditions in order to facilitate a direct comparison of the absolute 2PA cross sections in the range 3.5-6.0 eV. For comparison, the theoretical 2PA cross sections are computed using the equation-of-motion coupled-cluster method with single and double substitutions. Simulated 2PA spectra based on the calculations reproduce the main features of the experimental spectra in solution, although the quantitative comparison is complicated by a number of uncertainties, including limitations of the theoretical model, vibronic structure, broadening of the experimental spectra, and solvent effects. The systematic comparison of experimental and theoretical spectra for this series of structurally similar compounds provides valuable insight into the nature of 2PA transitions in conjugated molecules. Notably, the orbital character and symmetry-based selection rules provide a foundation for interpreting the features of the experimental 2PA spectra in unprecedented detail. Published by AIP Publishing. [http://dx.doi.org/10.1063/1.4979651]
\end{abstract}

\section{INTRODUCTION}

The development of new two-photon active compounds has enabled rapid and widespread advances in multi-photon techniques, including fluorescence imaging and microscopy, 3D fabrication, optical data storage, photodynamic therapy, and spatially controlled chemical activation. ${ }^{1-16}$ Sustaining and enhancing the current level of progress in these areas requires novel chromophores with favorable two-photon absorption (2PA) properties. ${ }^{17-19}$ An essential ingredient in developing such compounds is the ability to predict and interpret experimental 2PA spectra using computational chemistry. Advances in computational methods now make such comparisons feasible, ${ }^{20-23}$ yet there has been little work so far to make quantitative comparisons of wavelength-dependent experimental and theoretical 2PA cross sections. These comparisons are important for benchmarking the calculations as well as providing insight into the fundamental features that determine non-linear optical properties of molecules and molecular aggregates.

Accurate 2PA spectra are difficult to measure experimentally and challenging to calculate using $a b$ initio theory. The experiments are plagued by weak signals, competing contributions from scattering and other nonlinear energy loss

\footnotetext{
a) Present address: Savannah River National Laboratory, Aiken, South Carolina 29808, USA.

b) Authors to whom correspondence should be addressed. Electronic addresses: krylov@usc.edu, elles@ku.edu.
}

processes, uncertainty in laser beam profiles, and, ultimately, difficulties in interpreting the often broad, overlapping bands of a 2PA spectrum. Calculations are complicated not only by the need to obtain accurate transition energies and intensities for molecules of reasonable size, but also the difficulty of including vibronic effects, solvent shifts, and other corrections to match the experimental situation. To address these issues, we compare the experimental and theoretical 2PA spectra for three aromatic compounds that differ primarily in molecular symmetry: trans-stilbene, cis-stilbene, and phenanthrene. These three well-studied molecules serve as a convenient series for comparing experimental and computational 2PA cross sections of structurally related compounds belonging to different point groups $\left(\mathrm{C}_{2 h}\right.$ for trans-stilbene, $\mathrm{C}_{2}$ for cis-stilbene, and $\mathrm{C}_{2 v}$ for phenanthrene).

The excited-state structure and dynamics of stilbene and phenanthrene have been studied extensively. ${ }^{24-27}$ Stilbene is a prototypical photochromic molecule that undergoes reversible photoisomerization and photocyclization reactions that are typical of many molecular switches. ${ }^{28-32}$ Whereas trans-stilbene photoisomerizes to cis-stilbene, the reverse isomerization reaction competes with electrocyclization of cis-stilbene to 4a,4b-dihydrophenanthrene, which irreversibly oxidizes to phenanthrene. ${ }^{25}$ Numerous excited-state calculations at various levels of theory have been reported for trans-stilbene, ${ }^{26,33-48}$ cis-stilbene, ${ }^{26,38,39,41-47,49,50}$ and phenanthrene, ${ }^{51-53}$ with some of the most comprehensive work by Merchan and coworkers using CASPT2 (complete active space self-consistent field with second-order perturbative 
correction). ${ }^{48,50,53}$ However, there have been only a limited number of studies on the 2PA properties, and the reported cross sections vary by orders of magnitude. Such inconsistencies and the difficulty of comparing results from different levels of theory, including different state ordering, highlight the need for a single set of computational results for comparison with the experimental 2PA spectra of all three compounds.

In this paper, we compare our experimentally measured broadband 2PA spectra with the spectra computed using the equation-of-motion coupled-cluster method for excited states with single and double substitutions (EOM-EE-CCSD). ${ }^{23}$ EOM-CC methods are capable of describing multiple electronic states, including electronically excited states that have multi-configurational wave functions. ${ }^{54-60}$ The calculated transition energies and intensities provide an important reference for modeling and analyzing the experimental spectra. We also analyze the electronic structure of the states that give rise to the dominant features in the $2 \mathrm{PA}$ spectra using wave function analysis tools. ${ }^{61-63}$

Measuring the broadband 2PA spectra back-to-back reduces uncertainties of the experimental parameters between measurements and provides a consistent set of spectra for comparison with theory. The experimental benchmarks include the transition energies, absolute 2PA cross sections, and polarization ratios. ${ }^{23,64}$ The theoretical approach and computational methodology are described in detail in a separate paper, ${ }^{65}$ where we also discuss general trends in 2PA spectra and analyze symmetry-based selection rules and molecular-orbital characters of the states giving rise to the most prominent $2 \mathrm{PA}$ transitions. Here, we give a concise summary of methodology and focus on the experimentally accessible region of the solution-phase spectrum (below $\sim 6.5 \mathrm{eV}$ ).

\section{METHODS}

\section{A. Experimental details}

We use a broadband pump-probe technique to measure the experimental 2PA spectra of trans-stilbene, cis-stilbene, and phenanthrene in solution. Details of the broadband 2PA measurement have been discussed previously ${ }^{66-70}$ and are described here only briefly. The 2PA spectra are measured by spatially and temporally overlapping a non-resonant pump pulse with a broadband probe pulse within a $1 \mathrm{~mm}$ path length quartz cuvette containing the sample. The attenuation of the probe is proportional to the (non-degenerate) two-photon absorption cross section $\sigma^{2 P A}$ at the total energy of one pump and one probe photon. Importantly, neither photon is absorbed alone. The 2PA cross sections are measured in GM (GöppertMayer, $1 \mathrm{GM}=10^{-50} \mathrm{~cm}^{4} \cdot \mathrm{s} \cdot$ molecule $^{-1} \cdot$ photon $^{-1}$ ), based on the wavelength-dependent depletion rate of the broadband probe field in the presence of the pump field.

The pump and probe pulses are derived from an ultrafast Ti:sapphire laser, using an optical parametric amplifier to generate tunable pump pulses in the visible-UV, and white-light continuum generation as the source of broadband probe pulses. Focusing a small fraction of the $800 \mathrm{~nm}$ fundamental into a 1 $\mathrm{cm}$ quartz cuvette filled with water produces stable white-light continuum probe pulses covering the range 750-350 nm (1.65$3.54 \mathrm{eV}){ }^{71,72} \mathrm{We}$ focus the pump and probe beams to $\sim 200$ and $\sim 70 \mu \mathrm{m}$ diameter, respectively, at the sample, where the average pump energy is usually $\sim 100 \mathrm{~nJ} /$ pulse. An optical chopper wheel blocks every other pump pulse before the sample for active background subtraction, and we control the relative orientation of the linearly polarized pump and probe light with a $\lambda / 2$ waveplate in the pump beam. After the sample, the transmitted probe beam is dispersed through a transmission grating onto a 256-element photodiode array for shot-to-shot detection. The 2PA measurements have a resolution of $\sim 0.14 \mathrm{eV}$, limited by the convolution of the pump pulse bandwidth and the resolution of the spectrograph.

The samples for the 2PA measurements are $0.5 \mathrm{M}$ solutions of trans-stilbene, cis-stilbene, or phenanthrene (all from Sigma-Aldrich, >96\%) in chloroform (Sigma-Aldrich, $\geq 99 \%$ ). We measure the 1PA spectra of more dilute solutions using a standard UV-vis spectrometer with a resolution of $\sim 0.03 \mathrm{eV}$. We measure 1PA spectra in both chloroform and cyclohexane, but report only the latter because that solvent has a larger optical bandgap. The 1PA spectra are nearly identical in the two solvents. ${ }^{73}$

\section{B. Computational details}

For exact wave functions, the two-photon transition moments at frequencies $\omega_{1}$ and $\omega_{2}$ between states $|0\rangle$ and $|k\rangle$ are given by the following expressions:

$$
\begin{aligned}
& M_{b c}^{k \leftarrow 0}=-\sum_{n}\left(\frac{\left\langle k\left|\hat{\mu}^{c}\right| n\right\rangle\left\langle n\left|\hat{\mu}^{b}\right| 0\right\rangle}{\Omega_{n 0}-\omega_{1}}+\frac{\left\langle k\left|\hat{\mu}^{b}\right| n\right\rangle\left\langle n\left|\hat{\mu}^{c}\right| 0\right\rangle}{\Omega_{n 0}-\omega_{2}}\right), \\
& M_{b c}^{0 \leftarrow k}=-\sum_{n}\left(\frac{\left\langle 0\left|\hat{\mu}^{b}\right| n\right\rangle\left\langle n\left|\hat{\mu}^{c}\right| k\right\rangle}{\Omega_{n 0}-\omega_{1}}+\frac{\left\langle 0\left|\hat{\mu}^{c}\right| n\right\rangle\left\langle n\left|\hat{\mu}^{b}\right| k\right\rangle}{\Omega_{n 0}-\omega_{2}}\right),
\end{aligned}
$$

where $\hat{\mu}$ denotes the dipole moment operator, $\Omega_{n 0}$ is the transition energy between states $|0\rangle$ and $|n\rangle$, and the sums run over all electronic states of the system. Following the expectationvalue approach to molecular properties, ${ }^{74}$ this expression is converted into a tractable form for computing two-photon moments for approximate wave functions by introducing auxiliary response-like equations. The application of this strategy to the EOM-CCSD wave functions is described in Ref. 23. Here, we calculate 2PA cross sections using this methodology, ${ }^{23}$ following the same protocol as in Ref. 65 . Note that, although the programmable expressions for the two-photon transition moments have different forms from the above sumover-state equations, they are formally and numerically identical. Thus, we will use Eqs. (1) and (2) for the analysis of the computed spectra.

From the two-photon transition moments, the components of the transition strength matrix, $S_{a b, c d}$, are computed as follows:

$$
S_{a b, c d}=0.5 *\left(M_{a b}^{0 \leftarrow k} M_{c d}^{k \leftarrow 0}+M_{c d}^{0 \leftarrow k} M_{a b}^{k \leftarrow 0}\right) .
$$

Using atomic units, the rotationally averaged 2PA strength, $\left\langle\delta^{2 P A}\right\rangle$, reads $^{75,76}$

$$
\left\langle\delta^{2 P A}\right\rangle=\frac{F}{30} \sum_{a, b} S_{a a, b b}+\frac{G}{30} \sum_{a, b} S_{a b, a b}+\frac{H}{30} \sum_{a, b} S_{a b, b a},
$$

where $F, G$, and $H$ are integer constants, which depend on the polarization of the incident light. $F=G=H=2$ for parallel 
linearly polarized light, whereas $F=-1, G=4, H=-1$ for perpendicular linearly polarized light. ${ }^{75}$ The macroscopic 2PA cross section $\left(\sigma^{2 P A}\right)$ in $\mathrm{GM}$ units is related to the molecular transition strength in the case of $\omega_{1}=\omega_{2}=\frac{E_{\text {exc }}}{2}$ through the following expression: ${ }^{64}$

$$
\sigma^{2 P A}=\frac{\pi^{3} \alpha a_{0}^{5}(2 \omega)^{2}}{c}\left\langle\delta^{2 P A}\right\rangle S\left(2 \omega, \omega_{0}, \Gamma\right),
$$

where $\alpha$ is the fine structure constant, $a_{0}$ is the Bohr radius, $\omega$ is the photon energy for excitation of $2 \omega, c$ is the speed of light, and $S\left(2 \omega, \omega_{0}, \Gamma\right)$ is the line-shape function describing spectral broadening effects. When two different frequencies are used, the macroscopic cross section is

$$
\sigma^{2 P A}=\frac{2 \pi^{3} \alpha a_{0}^{5} \omega_{\sigma}^{2}}{c}\left\langle\delta^{2 P A}\right\rangle S\left(\omega_{\sigma}, \omega_{0}, \Gamma\right),
$$

where $\omega_{\sigma}=\omega_{1}+\omega_{2}$.

The line-shape function introduces a phenomenological broadening for the computed stick spectrum to account for spectral broadening due to rovibrational excitations and collisional dynamics. Using a different broadening factor affects not only the width of each peak, but also the maximum amplitude. The difficulties of computing broadening factors from the first-principles limit the predictive power of the calculations. Here, we use fitted experimental damping factors to facilitate the direct comparison of the computed spectra with experiment and to illustrate the impact of varying damping factors on the spectra. For calibration purposes, we also calculated 1PA spectra. We used $\Gamma=0.4 \mathrm{eV}$ for cis-stilbene and $\Gamma=0.2$ $\mathrm{eV}$ for trans-stilbene and phenanthrene to best reproduce the experimental results. In contrast to the calculations reported in Ref. 65, here we compute 2PA spectra for the non-degenerate case $\left(\omega_{2}=E_{e x}-\omega_{1}\right)$, to match the experimental conditions, and also consider Franck-Condon and solvent contributions to the 2PA spectra. We also compare the experimental and computed polarization ratios, which provides additional validation of the spectral assignments.

The structures of trans-stilbene $\left(C_{2 h}\right)$, cis-stilbene $\left(C_{2}\right)$, and phenanthrene $\left(C_{2 v}\right)$ are from Ref. 65 , where they were optimized at the B3LYP/cc-pVTZ level of theory. Here we employ the d-aug-cc-pVDZ Dunning's basis set from which the most diffuse set of $d$-functions was removed. We also employ the Cholesky decomposition of the two-electron integrals ${ }^{23,77}$ with a threshold of $10^{-2}$ and freeze core electrons. The basis-sets effects and the impact of Cholesky decomposition have been investigated in detail in Refs. 23 and 65. It was shown (for ethylene) that for 2PA transitions with energies below $\sim 10.5 \mathrm{eV}$, the d-aug-cc-pVDZ basis set is able to reproduce intensities obtained with the d-aug-cc-pVTZ basis set. The difference between excitation energies computed with the two bases was $\leq 1.6 \%$. For the two-photon transitions above $10.5 \mathrm{eV}$, at least a triple- $\zeta$ basis set is needed. Singly augmented basis sets are not sufficient, but the second set of diffuse $d$-functions from the doubly augmented set are only needed for high energy states with energies above $\sim 9 \mathrm{eV}$. Similar behavior was observed for toluene, confirming that $\mathrm{d}-\mathrm{aug}(-\mathrm{d})$-cc-pVDZ basis set is sufficient.

All calculations were performed using the Q-Chem electronic structure program. ${ }^{78,79}$ The reported symmetry labels of the electronic states and MOs correspond to the standard molecular orientation used in Q-Chem, which differs for some irreducible representations from the Mulliken convention. ${ }^{80,81}$ Additional details are provided in the supplementary material.

\section{RESULTS AND DISCUSSION}

We begin by briefly summarizing relevant findings of Ref. 65. By applying symmetry-imposed selection rules, we showed that fully symmetric transitions typically dominate the 2PA spectra for parallel polarization, because the diagonal tensor elements of the two-photon transition matrix belong to the totally symmetric irreps. Similar symmetry arguments based on the atomic selection rules predict that $\Delta l=0$ transitions have the largest 2PA cross sections among Rydberg-like transitions, which helped to rationalize the calculated 2PA spectra of ethylene, toluene, stilbenes, and phenanthrene. ${ }^{65}$ The analysis of the wave functions has revealed that, for the same molecule, a more diffuse character of the target state corresponds to a larger 2PA cross section, which can be explained by considering transition dipole moments between the molecular orbitals involved in the brightest valence transitions and the diffuse Rydberg orbitals. In agreement with previous computational studies, we found ${ }^{65}$ that trans-stilbene has the largest 2PA cross sections, as compared to cis-stilbene and phenanthrene due to the most extensive delocalization of the relevant states. The $5^{1} \mathrm{~A}_{g}$ state, which was found to have the largest cross section, can be described as $\pi \rightarrow p$-like Rydberg transition, where the diffuse natural transition orbitals (NTOs) of the electron resemble the bonding linear combination of $p$-like Rydberg MOs on each phenyl ring. Due to the nonplanar geometry, the exciton delocalization is reduced in cis-stilbene, resulting in lower 2PA cross sections ( $\pi \rightarrow 6^{1} \mathrm{~A}$ and $9^{1} \mathrm{~A}$ ) relative to the $5^{1} \mathrm{~A}_{g}$ state of trans-stilbene. In phenanthrene, the largest 2PA cross sections correspond to $p$ - and $d$-like Rydberg target states. While in Ref. 65 we characterized the most prominent 2PA transitions, here we focus on a lower-energy part of the spectra, which we also investigated experimentally.

\section{A. Comparison of experimental 1PA and 2PA spectra}

Figure 1 compares the experimental 1PA and 2PA spectra for trans-stilbene, cis-stilbene, and phenanthrene. All three compounds have distinctly different absorption spectra for one- versus two-photon excitation. For example, the linear absorption spectrum of trans-stilbene has absorption bands near 4.1, 5.4, and $6.1 \mathrm{eV}$ (typically labeled A, B, and C), whereas the 2PA spectrum of the same molecule has a weak shoulder near $4.3 \mathrm{eV}$, a stronger band centered near $5.1 \mathrm{eV}$, and a very intense band at higher energy. The higher-energy band was previously observed to have a maximum near $6.4 \mathrm{eV}$ (Ref. 66). The stark difference between the 1PA and 2PA spectra of trans-stilbene is a consequence of the symmetry selection rules that formally restrict ungerade transitions to the 1PA spectrum and gerade transitions to the 2PA spectrum. Although cis-stilbene and phenanthrene are noncentrosymmetric, those compounds also have very different 1PA and 2PA spectra, highlighting the ability to derive complementary information from the two spectroscopic methods. 

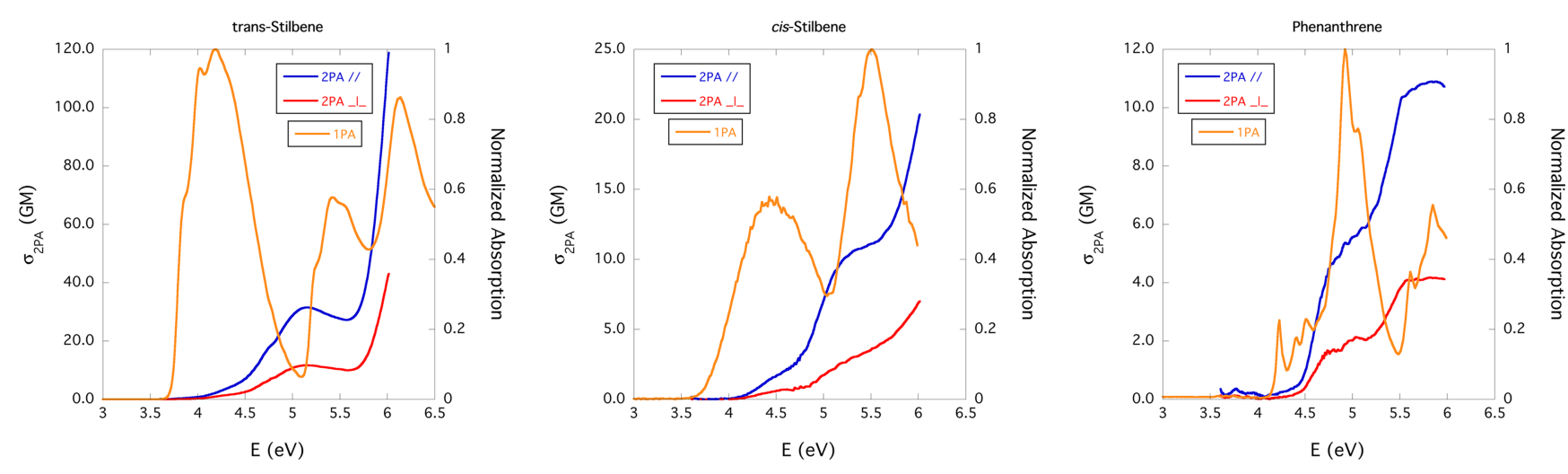

FIG. 1. Experimental 1PA and 2PA spectra of trans-stilbene, cis-stilbene, and phenanthrene.

An interesting difference between the experimental 1PA and 2PA spectra in Figure 1 is the degree of vibronic structure. The 1PA spectrum of phenanthrene has the most clearly resolved structure, which is typical of rigid, planar molecules. ${ }^{51}$ Although not as sharply defined as phenanthrene, the 1PA bands of trans-stilbene also have some vibrational structure. However, we do not observe any vibronic structure in the 2PA spectra, with the possible exception of the very weak band below $4 \mathrm{eV}$ in the spectrum of phenanthrene. The 2PA measurements have a resolution of $\sim 0.14 \mathrm{eV}$, which is sufficient to resolve a structure similar to that observed in the linear spectra. In contrast with other compounds, cis-stilbene has no discernible vibronic structure. Both the 1PA and 2PA bands of cis-stilbene may be broadened to a larger extent than in other compounds due to the shallower potential and nonplanar geometry of the molecule in the ground state giving a wider range of structural conformations.

The experimental 2PA transition energies and absolute cross sections of all three compounds are summarized in Table I. The reported confidence limits in the table are an estimate of the uncertainty in the absolute 2PA cross section at each energy. The relative cross sections within a spectrum are more precise, because we simultaneously measure the cross section at each energy using the broadband 2PA technique. The primary source of uncertainty in these measurements is the spatial overlap of pump and probe beams; ${ }^{82}$ therefore, we make back-to-back measurements under identical conditions in order to facilitate the comparison between compounds. Previously reported cross sections vary by orders of magnitude and have never been measured consistently for the set of all three compounds. ${ }^{66}$

TABLE I. Summary of experimental 2PA transition energies and cross sections. $^{\text {a }}$

\begin{tabular}{|c|c|c|c|c|c|}
\hline \multicolumn{2}{|c|}{ trans-stilbene } & \multicolumn{2}{|c|}{ cis-stilbene } & \multicolumn{2}{|c|}{ Phenanthrene } \\
\hline $\mathrm{E}_{e x}(\mathrm{eV})$ & $\sigma^{2 P A}(\mathrm{GM})$ & $\mathrm{E}_{e x}(\mathrm{eV})$ & $\sigma^{2 P A}(\mathrm{GM})$ & $\mathrm{E}_{e x}(\mathrm{eV})$ & $\sigma^{2 P A}(\mathrm{GM})$ \\
\hline 4.3 & $4.3 \pm 1.8$ & 4.4 & $1.3 \pm 0.6$ & 3.8 & $0.4 \pm 0.2$ \\
\hline 5.1 & $32 \pm 13$ & 5.2 & $10 \pm 4$ & 4.8 & $4.7 \pm 1.9$ \\
\hline 6.4 & $270 \pm 110^{\mathrm{b}}$ & & & 5.5 & $10 \pm 4$ \\
\hline
\end{tabular}

$\overline{{ }^{a} \text { Uncertainties are estimated } 95 \% \text { confidence limits for the absolute 2PA cross sections. }}$ Relative cross sections within each spectrum are known to have a much better precision. ${ }^{\mathrm{b}}$ From Ref. 66.
Figure 1 shows the 2PA spectra for parallel $\left(\sigma_{\|}^{2 P A}\right)$ and perpendicular $\left(\sigma_{\perp}^{2 P A}\right)$ relative polarization of the pump and probe beams. The relative intensity of the parallel and perpendicular transitions for a given 2PA band depends on the symmetry of the transition through the transition dipole tensor. ${ }^{75,76,83}$ For an orientationally averaged sample, the polarization ratio $r=\sigma_{\|}^{2 P A} / \sigma_{\perp}^{2 P A}$ is limited to $r \geq 4 / 3$ for totally symmetric transitions and $r \leq 4 / 3$ for non-totally symmetric transitions. The spectra for parallel polarization are more than 2-3 times more intense than the perpendicular spectra across the entire range of 2PA energies for each molecule, indicating that twophoton transitions in this energy range primarily access totally symmetric excited states. The prevalence of totally symmetric transitions in 2PA spectra can be rationalized by analyzing the sum-over-states expression of the total cross section in the context of symmetry-based selection rules. ${ }^{65} \mathrm{We}$ describe the polarization dependence of the 2PA spectra in more detail below.

\section{B. Comparing theory and experiment: One-photon absorption}

Before examining the 2PA spectroscopy of the target compounds, it is instructive to compare the experimental and theoretical 1PA spectra. The calculated 1PA energies and oscillator strengths (obtained at the EOM-EE-CCSD/d-aug(d)-cc-pVDZ level of theory) are listed in Tables II-IV. We calculate transition energies for the lowest 8 excited states of each symmetry type. For comparison with experiment, we also apply a Gaussian broadening factor of $0.2-0.4 \mathrm{eV}$ to each transition in the calculated spectrum. The tables show the maximum extinction coefficients resulting from the broadening of each transition. Figure 2 shows both the stick and broadened spectra for each compound, along with the experimental spectra. Vibronic effects play an important role in determining the exact shapes of the experimental spectra, but the general features of all three 1PA spectra are reproduced grosso modo by simply applying the phenomenological broadening and shifting the calculated spectra to lower energy by $0.4-0.7 \mathrm{eV}$.

The strongest transition in the low-energy region of each spectrum is due to $\pi \rightarrow \pi^{*}$ excitation, as illustrated by the NTOs in Figure 3. The NTOs represent transitions to the $1^{1} \mathrm{~B}_{u}\left(\pi \pi^{*}\right)$ state of trans-stilbene, the $2^{1} \mathrm{~B}\left(\pi \pi^{*}\right)$ state of 
TABLE II. Term labels, excitation energies $\left(\mathrm{E}_{e x}\right)$, oscillator strengths $\left(\mathrm{f}_{l}\right)$, extinction coefficients $(\varepsilon(\omega))$, microscopic $\left(\left\langle\delta_{\|}^{2 P A}\right\rangle\right)$ and macroscopic $\left(\sigma_{\|}^{2 P A}\right) 2 \mathrm{PA}$ cross sections, and polarization ratios of trans-stilbene. A pump wavelength of $380 \mathrm{~nm}$ has been used for 2PA calculations. EOM-EE-CCSD/daug(-d)-cc-pVDZ.

\begin{tabular}{|c|c|c|c|c|c|c|}
\hline States & $\mathrm{E}_{e x}(\mathrm{eV})$ & $\mathrm{f}_{L}$ & $\varepsilon(\omega)\left(\mathrm{L} \mathrm{mol}^{-1} \mathrm{~cm}^{-1}\right)$ & $\left\langle\delta_{\|}^{2 P A}\right\rangle$ (a.u.) & $\sigma_{\|}^{2 P A}(\mathrm{GM})$ & $r=\sigma_{\|}^{2 P A} / \sigma_{\perp}^{2 P A}$ \\
\hline trans-stilbene & & & $\Gamma=0.2 \mathrm{eV}$ & & $\Gamma=0.1 \mathrm{eV}$ & \\
\hline $2^{1} \mathrm{~A}_{g}$ & 4.81 & 0 & 0 & 239 & 5.97 & 1.47 \\
\hline $3^{1} \mathrm{~A}_{g}$ & 5.74 & 0 & 0 & 1182 & 42.09 & 2.66 \\
\hline $4^{1} \mathrm{~A}_{g}$ & 6.15 & 0 & 0 & 4070 & 166.29 & 2.62 \\
\hline $5^{1} \mathrm{~A}_{g}$ & 6.28 & 0 & 0 & 41357 & 1765.33 & 3.07 \\
\hline $6^{1} \mathrm{~A}_{g}$ & 6.45 & 0 & 0 & 27 & 1.21 & 1.25 \\
\hline $7^{1} \mathrm{~A}_{g}$ & 6.68 & 0 & 0 & 376 & 18.23 & 1.53 \\
\hline $8^{1} \mathrm{~A}_{g}$ & 6.77 & 0 & 0 & 159 & 7.87 & 1.86 \\
\hline $9^{1} \mathrm{~A}_{g}$ & 6.92 & 0 & 0 & 88 & 4.55 & 10.2 \\
\hline $1^{1} \mathrm{~B}_{g}$ & 5.53 & 0 & 0 & 11 & 0.37 & 0.93 \\
\hline $2^{1} \mathrm{~B}_{g}$ & 5.67 & 0 & 0 & 172 & 5.96 & 1.33 \\
\hline $3^{1} \mathrm{~B}_{g}$ & 6.38 & 0 & 0 & 119 & 5.21 & 1.33 \\
\hline $4^{1} \mathrm{~B}_{g}$ & 6.40 & 0 & 0 & 101 & 4.46 & 1.33 \\
\hline $5^{1} \mathrm{~B}_{g}$ & 6.58 & 0 & 0 & 91 & 4.28 & 1.33 \\
\hline $6^{1} \mathrm{~B}_{g}^{8}$ & 6.65 & 0 & 0 & 8 & 0.37 & 1.33 \\
\hline $7^{1} \mathrm{~B}_{g}^{8}$ & 6.72 & 0 & 0 & 14 & 0.70 & 1.33 \\
\hline $8^{1} \mathrm{~B}_{g}$ & 6.90 & 0 & 0 & 20 & 1.03 & 1.19 \\
\hline $1^{1} \mathrm{~A}_{u}$ & 5.27 & 0.001 & 100 & 0 & 0 & $0 / 0$ \\
\hline $2^{1} \mathrm{~A}_{u}$ & 5.98 & 0.010 & 795 & 0 & 0 & $0 / 0$ \\
\hline $3^{1} \mathrm{~A}_{u}$ & 6.11 & 0.000 & 24 & 0 & 0 & $0 / 0$ \\
\hline $4^{1} \mathrm{~A}_{u}$ & 6.14 & 0.002 & 154 & 0 & 0 & $0 / 0$ \\
\hline $5^{1} \mathrm{~A}_{u}$ & 6.46 & 0.000 & 3 & 0 & 0 & $0 / 0$ \\
\hline $6^{1} \mathrm{~A}_{u}$ & 6.52 & 0.000 & 40 & 0 & 0 & $0 / 0$ \\
\hline $7^{1} \mathrm{~A}_{u}$ & 6.78 & 0.004 & 328 & 0 & 0 & $0 / 0$ \\
\hline $8^{1} \mathrm{~A}_{u}$ & 6.83 & 0.000 & 18 & 0 & 0 & $0 / 0$ \\
\hline $1^{1} \mathrm{~B}_{u}$ & 4.55 & 0.712 & 57674 & 0 & 0 & $0 / 0$ \\
\hline $2^{1} \mathrm{~B}_{u}$ & 4.85 & 0.311 & 25219 & 0 & 0 & $0 / 0$ \\
\hline $3{ }^{1} \mathrm{~B}_{u}$ & 6.11 & 0.326 & 26412 & 0 & 0 & $0 / 0$ \\
\hline $4^{1} \mathrm{~B}_{u}$ & 6.26 & 0.059 & 4739 & 0 & 0 & $0 / 0$ \\
\hline $5^{1} \mathrm{~B}_{u}$ & 6.46 & 0.183 & 14830 & 0 & 0 & $0 / 0$ \\
\hline $6^{1} \mathrm{~B}_{u}$ & 6.81 & 0.568 & 46019 & 0 & 0 & $0 / 0$ \\
\hline $7^{1} \mathrm{~B}_{u}$ & 7.07 & 0.018 & 1453 & 0 & 0 & $0 / 0$ \\
\hline $8^{1} \mathrm{~B}_{u}$ & 7.16 & 0.073 & 5910 & 0 & 0 & $0 / 0$ \\
\hline
\end{tabular}

cis-stilbene, and the $3{ }^{1} \mathrm{~B}_{1}\left(\pi \pi^{*}\right)$ state of phenanthrene. A single NTO pair represents the transitions for trans-stilbene and cis-stilbene, but two pairs of NTOs are required for phenanthrene. In each case, the NTOs confirm that these optically bright $\pi \pi^{*}$ states have primarily a valence (rather than Rydberg) character. For all three molecules, transitions at higher energies tend to have a more diffuse, Rydberg-like character in the gas-phase calculations. ${ }^{65}$

Several factors contribute to the calculated transitions being shifted to higher energy relative to the experimental spectra. First, there are intrinsic limitations of the theoretical approach, including neglected higher-order excitations and limited basis sets. Typical error bars for states with a singly excited character are in the range of $0.3 \mathrm{eV}$ for EOM-EECCSD, which includes only single and double excitations. The intrinsic errors due to missing triple (and higher) excitations usually give energies that are higher than the experimental values. ${ }^{84-86}$ Insufficient one-electron basis sets might lead to additional errors, especially for higher-lying electronic states that have more diffuse character.

The second important source of uncertainty is the solvent. Figure 2 shows the experimental 1PA spectra in cyclohexane, but the EOM-CCSD calculations do not include any solvent effects. While solvatochromic shifts are usually small for valence states in non-polar solvents, solvent effects could be more significant for higher-lying states that have a more diffuse character. We note that the fate of Rydberg states in solution is unclear, and even the sign of an anticipated solventinduced shift is not obvious for diffuse excited states. On one hand, Pauli repulsion between the diffuse wavefunction of an excited electron and the surrounding solvent may cause a blue shift due to confinement in solution, but, on the other hand, solvent stabilization of the cationic core may result in a red shift. $^{87}$

In order to estimate solvatochromic shifts relative to the gas phase, we examine solvation effects in the calculated 
TABLE III. Term labels, excitation energies $\left(\mathrm{E}_{e x}\right)$, oscillator strengths $\left(\mathrm{f}_{l}\right)$, extinction coefficients $(\varepsilon(\omega))$, microscopic $\left(\left\langle\delta_{\|}^{2 P A}\right\rangle\right)$ and macroscopic $\left(\sigma_{\|}^{2 P A}\right) 2 \mathrm{PA}$ cross sections, and polarization ratios of cis-stilbene. A pump wavelength of $380 \mathrm{~nm}$ has been used for 2PA calculations. EOM-EE-CCSD/daug(-d)-cc-pVDZ.

\begin{tabular}{|c|c|c|c|c|c|c|}
\hline States & $\mathrm{E}_{e x}(\mathrm{eV})$ & $\mathrm{f}_{L}$ & $\varepsilon(\omega)\left(\mathrm{L} \mathrm{mol}^{-1} \mathrm{~cm}^{-1}\right)$ & $\left\langle\delta_{\|}^{2 P A}\right\rangle$ (a.u.) & $\sigma_{\|}^{2 P A}(\mathrm{GM})$ & $r=\sigma_{\|}^{2 P A} / \sigma_{\perp}^{2 P A}$ \\
\hline cis-stilbene & & & $\Gamma=0.4 \mathrm{eV}$ & & $\Gamma=0.1 \mathrm{eV}$ & \\
\hline $2^{1} \mathrm{~A}$ & 4.95 & 0.000 & 1 & 171 & 4.52 & 2.23 \\
\hline $3^{1} \mathrm{~A}$ & 5.88 & 0.006 & 240 & 89 & 3.34 & 1.66 \\
\hline $4^{1} \mathrm{~A}$ & 5.94 & 0.012 & 467 & 85 & 3.26 & 1.39 \\
\hline $5^{1} \mathrm{~A}$ & 6.19 & 0.043 & 1728 & 1169 & 48.60 & 3.42 \\
\hline $6^{1} \mathrm{~A}$ & 6.26 & 0.000 & 11 & 3812 & 161.32 & 3.17 \\
\hline $7^{1} \mathrm{~A}$ & 6.35 & 0.029 & 1165 & 589 & 25.56 & 8.42 \\
\hline $8^{1} \mathrm{~A}$ & 6.45 & 0.000 & 8 & 147 & 6.63 & 1.58 \\
\hline $9^{1} \mathrm{~A}$ & 6.50 & 0.000 & 10 & 3126 & 142.85 & 4.30 \\
\hline $1^{1} \mathrm{~B}$ & 4.81 & 0.009 & 347 & 4 & 0.10 & 1.32 \\
\hline $2^{1} \mathrm{~B}$ & 4.91 & 0.445 & 18014 & 4 & 0.09 & 1.33 \\
\hline $3{ }^{1} \mathrm{~B}$ & 5.48 & 0.021 & 842 & 6 & 0.18 & 1.29 \\
\hline $4^{1} \mathrm{~B}$ & 5.84 & 0.002 & 101 & 74 & 2.75 & 1.33 \\
\hline $5^{1} \mathrm{~B}$ & 6.17 & 0.302 & 12241 & 17 & 0.72 & 1.33 \\
\hline $6^{1} \mathrm{~B}$ & 6.32 & 0.069 & 2784 & 10 & 0.44 & 1.33 \\
\hline $7^{1} \mathrm{~B}$ & 6.38 & 0.006 & 241 & 64 & 2.79 & 1.33 \\
\hline $8^{1} \mathrm{~B}$ & 6.44 & 0.082 & 3308 & 3 & 0.14 & 1.25 \\
\hline
\end{tabular}

1PA spectrum of trans-stilbene using time-dependent density functional theory (TD-DFT) and a non-equilibrium solvation method (perturbation-theoretical state-specific + perturbationtheoretical linear-response type integral equation formulation of polarizable continuum model (ptSS + ptLR)IEFPCM) ${ }^{88,89}$ The $\omega$ B97X-D exchange-correlation functional with the d-aug(-d)-cc-pVDZ basis set gives good agreement with the calculated EOM-CCSD transition energies in vacuum (Fig. S1 of the supplementary material). $\omega \mathrm{B} 97$ and $\omega \mathrm{B} 97 \mathrm{X}$ functionals were also tested and yielded larger deviations from EOM-CCSD.

Table V compares the TD-DFT excitation energies and oscillator strengths of the three brightest states of transstilbene in vacuum, chloroform, and cyclohexane. The solvent shifts are almost identical for chloroform and cyclohexane, which is consistent with the experimental observation that the absorption spectra are the same in both solvents. ${ }^{73}$ Both solvents shift the excitation energy of the $1^{1} \mathrm{~B}_{u}$ state closer to the experimental value by $0.17 \mathrm{eV}$, but the $3^{1} \mathrm{~B}_{u}$ and $6^{1} \mathrm{~B}_{u}$ states shift to lower energy by only $0.08 \mathrm{eV}$. Although the discrepancy suggests that at least some higher-lying states may have smaller solvatochromic shifts, as we discuss in the context of the simulated 2PA spectra below, continuum solvation models cannot reliably predict solvation effects for Rydberg states (including the $3^{1} \mathrm{~B}_{u}$ and $6^{1} \mathrm{~B}_{u}$ states of trans-stilbene). Therefore, we can only conclude from the IEFPCM/TDDFT calculations that solvation red-shifts the low-lying valence states by about $0.2 \mathrm{eV}$.

The third reason for the discrepancy between experimental and theoretical spectra is the vibronic structure of the experimental absorption bands, which is only partially represented by the phenomenological broadening of the calculated spectra. While it is difficult to account for the vibronic structure in polyatomic molecules, it is instructive to consider the difference between adiabatic and vertical excitation energies. ${ }^{90}$ In polyatomic molecules, the maximum of the absorption often corresponds to adiabatic rather than vertical transitions, giving rise to an apparent red-shift of the bands. ${ }^{90}$ Below, we use a simple method ${ }^{91}$ for estimating the magnitude of vibronic effects in the 2PA spectra that circumvents costly excited-state geometry optimization calculations. Since Rydberg states can be described as a cationic core with an electron occupying a diffuse orbital, the structure and vibrational frequencies are usually quite similar to those of the respective cation..$^{91}$ Thus, we estimate excited-state relaxation effects by computing the difference between the total excited-state energies at the geometries of the neutral (FranckCondon structure) and the cationic species. This approach is applied to the evaluation of 2PA spectra below and may account for some of the discrepancy in the 1PA spectra as well. Non-Condon effects may also contribute small additional shifts. ${ }^{92,93}$

The net result of the effects described above is a systematic blue shift of the calculated gas-phase EOM-CCSD transition energies by about $0.5 \mathrm{eV}$ relative to the experimental values in solution, which accounts for most, if not all, of the observed shifts of $0.4-0.7 \mathrm{eV}$ in Figure 2. The extent of the shift depends on the electronic character of each transition, but similar shifts are expected for the 2PA bands, as discussed in Secs. III C 1-III C 5.

\section{Comparing theory and experiment: Two-photon absorption}

\section{Comparison between computed and experimental spectra}

Fig. 4 compares the experimental and theoretical 2PA spectra of trans-stilbene, cis-stilbene, and phenanthrene for parallel linear polarization. The experimental spectra were obtained by combining the results from broadband 
TABLE IV. Term labels, excitation energies $\left(\mathrm{E}_{e x}\right)$, oscillator strengths $\left(\mathrm{f}_{l}\right)$, extinction coefficients $(\varepsilon(\omega))$, microscopic $\left(\left\langle\delta_{\|}^{2 P A}\right\rangle\right)$ and macroscopic $\left(\sigma_{\|}^{2 P A}\right) 2 \mathrm{PA}$ cross sections, and polarization ratios of phenanthrene. A pump wavelength of $380 \mathrm{~nm}$ has been used for 2PA calculations. EOM-EE-CCSD/daug(-d)-cc-pVDZ.

\begin{tabular}{|c|c|c|c|c|c|c|}
\hline States & $\mathrm{E}_{e x}(\mathrm{eV})$ & $\mathrm{f}_{L}$ & $\varepsilon(\omega)\left(\mathrm{L} \mathrm{mol}^{-1} \mathrm{~cm}^{-1}\right)$ & $\left\langle\delta_{\|}^{2 P A}\right\rangle$ (a.u.) & $\sigma_{\|}^{2 P A}(\mathrm{GM})$ & $r=\sigma_{\|}^{2 P A} / \sigma_{\perp}^{2 P A}$ \\
\hline Phenanthrene & & & $\Gamma=0.2 \mathrm{eV}$ & & $\Gamma=0.1 \mathrm{eV}$ & \\
\hline $2^{1} \mathrm{~A}_{1}$ & 4.16 & 0.001 & 57 & 29 & 0.54 & 1.37 \\
\hline $3^{1} \mathrm{~A}_{1}$ & 5.55 & 0.123 & 9956 & 320 & 10.66 & 1.76 \\
\hline $4^{1} \mathrm{~A}_{1}$ & 5.85 & 0.163 & 13202 & 730 & 27.07 & 2.04 \\
\hline $5^{1} \mathrm{~A}_{1}$ & 6.11 & 0.000 & 26 & 2121 & 85.87 & 5.61 \\
\hline $6^{1} \mathrm{~A}_{1}$ & 6.32 & 0.120 & 9708 & 82 & 3.54 & 1.34 \\
\hline $7^{1} \mathrm{~A}_{1}$ & 6.54 & 0.001 & 66 & 22 & 1.02 & 3.54 \\
\hline $8^{1} \mathrm{~A}_{1}$ & 6.69 & 0.033 & 2698 & 464 & 22.45 & 1.33 \\
\hline $9^{1} \mathrm{~A}_{1}$ & 6.82 & 0.008 & 686 & 1793 & 90.35 & 4.08 \\
\hline $1^{1} \mathrm{~A}_{2}$ & 5.69 & 0 & 0 & 50 & 1.74 & 1.31 \\
\hline $2^{1} \mathrm{~A}_{2}$ & 5.86 & 0 & 0 & 37 & 1.36 & 1.33 \\
\hline $3^{1} \mathrm{~A}_{2}$ & 6.07 & 0 & 0 & 31 & 1.22 & 1.21 \\
\hline $4^{1} \mathrm{~A}_{2}$ & 6.27 & 0 & 0 & 56 & 2.35 & 1.19 \\
\hline $5^{1} \mathrm{~A}_{2}$ & 6.40 & 0 & 0 & 106 & 4.66 & 1.28 \\
\hline $6^{1} \mathrm{~A}_{2}$ & 6.54 & 0 & 0 & 96 & 4.41 & 0.78 \\
\hline $7^{1} \mathrm{~A}_{2}$ & 6.70 & 0 & 0 & 1 & 0.06 & 0.82 \\
\hline $8^{1} \mathrm{~A}_{2}$ & 6.80 & 0 & 0 & 9 & 0.47 & 0.52 \\
\hline $1^{1} \mathrm{~B}_{1}$ & 4.94 & 0.026 & 2120 & 15 & 0.40 & 1.33 \\
\hline $2^{1} \mathrm{~B}_{1}$ & 5.16 & 0.060 & 4862 & 61 & 1.76 & 1.33 \\
\hline $3^{1} \mathrm{~B}_{1}$ & 5.63 & 1.120 & 90723 & 2 & 0.06 & 0.01 \\
\hline $4^{1} \mathrm{~B}_{1}$ & 6.22 & 0.242 & 19600 & 7 & 0.31 & 1.21 \\
\hline $5^{1} \mathrm{~B}_{1}$ & 6.45 & 0.010 & 843 & 23 & 1.05 & 0.61 \\
\hline $6^{1} \mathrm{~B}_{1}$ & 6.65 & 0.105 & 8488 & 8 & 0.37 & 0.01 \\
\hline $7^{1} \mathrm{~B}_{1}$ & 6.80 & 0.008 & 678 & 0 & 0.01 & 0.02 \\
\hline $8^{1} \mathrm{~B}_{1}$ & 6.83 & 0.352 & 28467 & 44 & 2.23 & 0.94 \\
\hline $1^{1} \mathrm{~B}_{2}$ & 5.45 & 0.006 & 481 & 9 & 0.29 & 1.33 \\
\hline $2^{1} B_{2}$ & 5.75 & 0.003 & 232 & 193 & 6.89 & 0.17 \\
\hline $3^{1} \mathrm{~B}_{2}$ & 6.04 & 0.017 & 1344 & 2 & 0.09 & 0.47 \\
\hline $4^{1} \mathrm{~B}_{2}$ & 6.19 & 0.001 & 53 & 2 & 0.09 & 0.60 \\
\hline $5^{1} \mathrm{~B}_{2}$ & 6.28 & 0.003 & 229 & 2 & 0.07 & 1.16 \\
\hline $6^{1} \mathrm{~B}_{2}$ & 6.53 & 0.001 & 51 & 28 & 1.28 & 1.24 \\
\hline $7^{1} \mathrm{~B}_{2}$ & 6.70 & 0.002 & 164 & 11 & 0.53 & 0.99 \\
\hline $8^{1} \mathrm{~B}_{2}$ & 6.74 & 0.002 & 123 & 0 & 0.00 & 0.24 \\
\hline
\end{tabular}

measurements at three different pump wavelengths: $380 \mathrm{~nm}$ $(3.26 \mathrm{eV}), 420 \mathrm{~nm}(2.95 \mathrm{eV})$, and $780 \mathrm{~nm}(1.59 \mathrm{eV})$. Although the different resonance condition for each pump wavelength affects the amplitude of the measured 2PA cross section at a given energy, we ignore this small effect by taking the average value of the cross section in overlapping regions of
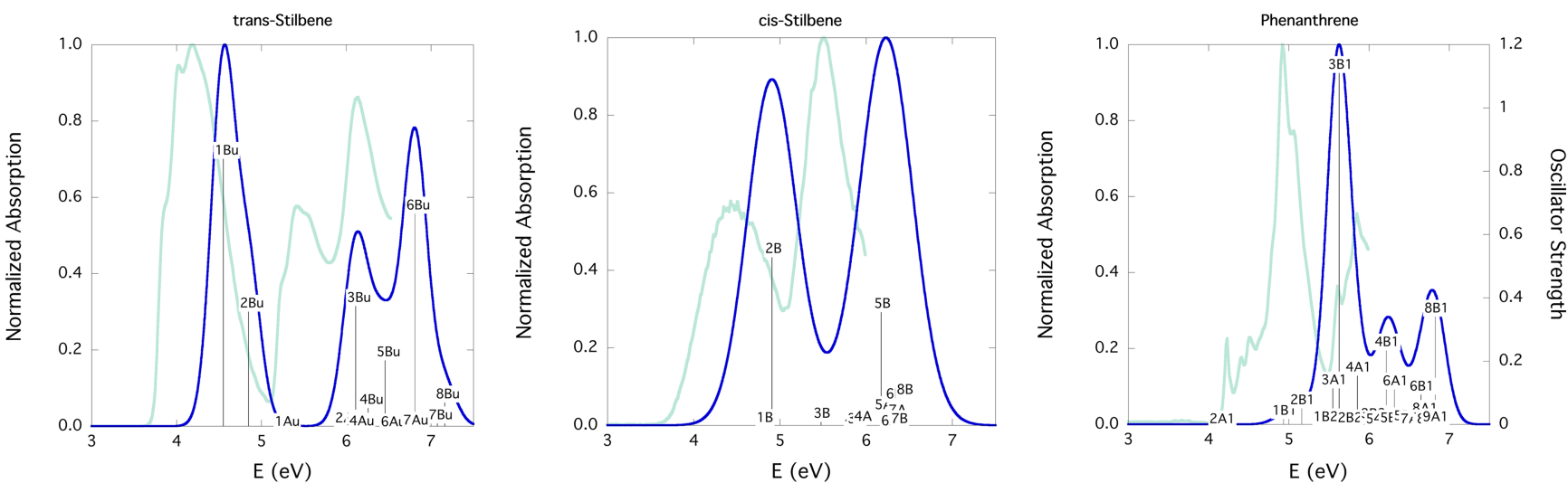

FIG. 2. Normalized experimental and theoretical 1PA spectra (light and dark blue lines, respectively) of trans-stilbene, $c$ is-stilbene, and phenanthrene. Theoretical spectra are from EOM-EE-CCSD/d-aug(-d)-cc-pVDZ with Gaussian broadening of $0.2 \mathrm{eV}$ for trans-stilbene and phenanthrene and $0.4 \mathrm{eV}$ for cis-stilbene. 


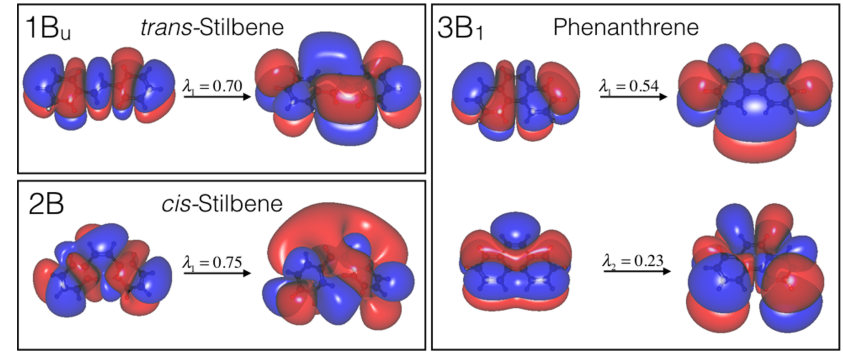

FIG. 3. NTOs corresponding to the $1{ }^{1} \mathrm{~B}_{u}, 2^{1} \mathrm{~B}$, and $3^{1} \mathrm{~B}_{1}$, excited states in trans-stilbene, cis-stilbene, and phenanthrene, respectively. The isovalue of $\sqrt{3 \cdot 10^{-6}}$ is used.

the spectrum..$^{73}$ The result is a single broadband 2PA spectrum that covers the full range of two-photon excitation energies (3.5-6.0 eV) for each compound. Similarly, the calculations are shown only for the $380 \mathrm{~nm}$ pump wavelength, although transition strengths were calculated for all three pump wavelengths. We calculate the first 8 states of each irreducible representation for trans-stilbene and cis-stilbene. In the case of phenanthrene, we are only able to calculate 2PA cross sections for the first five excited states of $A_{1}, A_{2}$, and $B_{1}$ symmetries, and the first six excited states of $\mathrm{B}_{2}$ symmetry, due to the one-photon resonance near $780 \mathrm{~nm}$.

The comparison between theoretical and experimental 2PA spectra is less straightforward than for the 1PA spectra. It is tempting to apply the same empirical shift and broadening factor for the 1PA and 2PA spectra. The result of this simple approach is shown in Figure S2 of the supplementary material, where we used $\Gamma=0.1 \mathrm{eV}$ for transstilbene and phenanthrene, $\Gamma=0.2 \mathrm{eV}$ for cis-stilbene, and applied a uniform red-shift of $0.7 \mathrm{eV}$ to each of the calculated 2PA transitions. The agreement between the experimental and simulated 2PA spectra is not as good as it is for the 1PA spectra. While the positions of many of the calculated transitions match the experimental bands reasonably well, the intensity profiles of the simulated spectra are quite different.

To properly compare the experimental and computed 2PA spectra, one needs to consider several issues. First, the most intense peaks in the calculated 2PA spectra correspond
TABLE V. Term labels, excitation energies $\left(\mathrm{E}_{e x}\right)$, and oscillator strengths $\left(\mathrm{f}_{l}\right)$ for the brightest 1PA states of trans-stilbene at the $\omega \mathrm{B} 97 \mathrm{XD} / \mathrm{d}$-aug(-d)cc-pVDZ level of theory, with and without solvent contributions computed by (ptSS + ptLR)IEFPCM.

\begin{tabular}{lcccccccc}
\hline \hline & \multicolumn{2}{c}{ Vacuum } & & \multicolumn{2}{c}{ Cyclohexane } & & \multicolumn{2}{c}{ Chloroform } \\
\cline { 2 - 3 } States & $\mathrm{E}_{e x}(\mathrm{eV})$ & $\mathrm{f}_{L}$ & & $\mathrm{E}_{e x}(\mathrm{eV})$ & $\mathrm{f}_{L}$ & & $\mathrm{E}_{e x}(\mathrm{eV})$ & $\mathrm{f}_{L}$ \\
\hline${ }^{1} \mathrm{~B}_{u} \mathrm{~B}_{u}$ & 4.38 & 1.177 & & 4.21 & 1.184 & & 4.21 & 1.189 \\
$3^{1} \mathrm{~B}_{u}$ & 6.10 & 0.432 & & 6.02 & 0.433 & & 6.03 & 0.436 \\
$6^{1} \mathrm{~B}_{u}$ & 6.75 & 0.610 & & 6.67 & 0.609 & & 6.67 & 0.594 \\
\hline \hline
\end{tabular}

to Rydberg states that might be strongly perturbed in solution. Second, the experimental peaks are very broad, which may hide many details, including contributions from excited states with small cross sections, vibronic effects, etc. Unfortunately, there is no systematic strategy for determining damping factors that match the experiment because the broadening may be different for each state; therefore, we begin by using a relatively small broadening factor of $0.1 \mathrm{eV}$ for all computed transitions, in order to prevent the dominant transitions from spilling over the entire spectrum. Note that using larger broadening factors results in lower peak intensities. Third, the macroscopic cross sections include an $\omega_{\sigma}^{2}$ dependence ( $\omega_{\sigma}$ is the transition energy) that increases the intensities of calculated bands that are shifted to higher excitation energies, and thus affects the overall shape of the spectrum. To address the frequency dependence of the 2PA cross sections, we divide the spectra by $\omega_{\sigma}^{2}$ in Fig. 5 and focus our attention on the experimentally accessible region of the spectrum below $\sim 6.5 \mathrm{eV}$. Note that dividing the experimental spectrum of phenanthrene by $\omega_{\sigma}^{2}$ reveals an abrupt drop of intensity above $5.5 \mathrm{eV}$. This is the region of the spectrum that was measured with $380 \mathrm{~nm}$ pump pulses and may be influenced by a (pre-)resonant excitation condition with the lowest 1PA band. Even after accounting for the $\omega_{\sigma}^{2}$ dependence of the 2PA spectrum, it is difficult to directly compare the calculated microscopic cross sections with the experimental results when using uniform (and arbitrary) damping factors. In order to address this issue, we deconvolute the experimental spectra in Sec. III C 2.
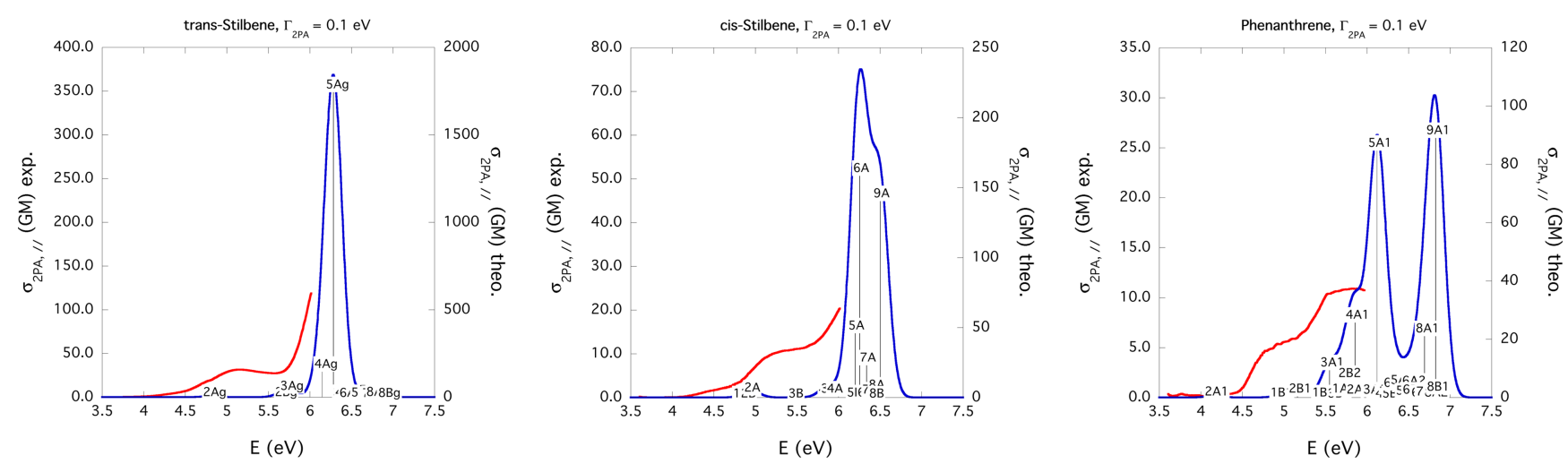

FIG. 4. Experimental (red) and theoretical (blue) 2PA spectra of trans-stilbene, cis-stilbene, and phenanthrene for parallel linear polarization. The theoretical spectra are obtained at the EOM-EE-CCSD/d-aug(-d)-cc-pVDZ level of theory with a Gaussian line shape and a damping factor of $0.1 \mathrm{eV}$. 

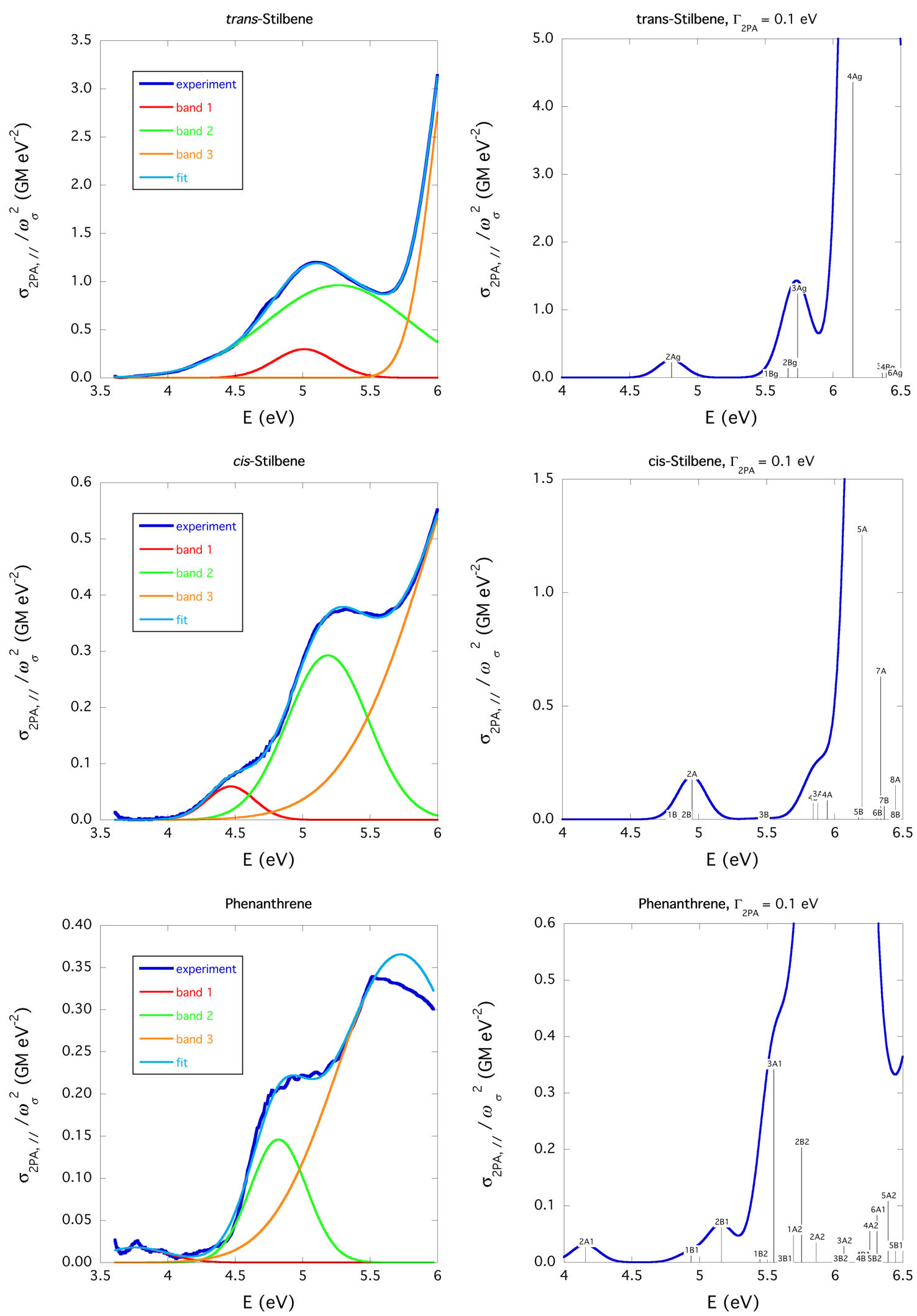

FIG. 5. Experimental and theoretical 2PA spectra for parallel polarization divided by $\omega_{\sigma}^{2}$. The thin grey lines are a deconvolution of each experimental spectrum using three Gaussian bands. Theoretical spectra (EOM-EE-CCSD/d-aug(-d)-cc-pVDZ) are broadened using a Gaussian line shape and a damping factor of $0.1 \mathrm{eV}$.

\section{Deconvolution and tentative assignment of the experimental spectra}

The purpose of the analysis presented in this section is to extract microscopic cross sections, $\left\langle\delta^{2 P A}\right\rangle$, and transition linewidths, $\Gamma$, associated with the strongest transitions in the experimental 2PA spectra. The deconvolution of the experimental 2PA spectra (after dividing by $\omega_{\sigma}^{2}$ ) uses a sum of Gaussian functions to represent the net macroscopic cross section as a function of the total (two-photon) excitation 
energy, $\omega_{\sigma}$,

$$
\frac{\sigma^{2 P A}}{\omega_{\sigma}^{2}}=\frac{2 \pi^{3} \alpha a_{0}^{5}}{c} \sum_{i}\left\langle\delta_{i}^{2 P A}\right\rangle \frac{\sqrt{\ln 2}}{\sqrt{\pi} \Gamma_{i}} \exp \left[-\ln 2\left(\frac{\omega_{\sigma}-\omega_{i}}{\Gamma_{i}}\right)^{2}\right]
$$

where $\omega_{i},\left\langle\delta_{i}^{2 P A}\right\rangle$, and $\Gamma_{i}$ are the fitting parameters associated with each transition. Fits to the experimental 2PA spectra are shown in Fig. 5. For each compound, we use a total of three Gaussian functions, which is the minimum number necessary to reproduce the general features of the spectra. These arbitrary fits are only meant to provide a preliminary interpretation of the experimental spectra, including an initial estimate of linewidths for simulating the spectra in Sec. III C 3. The excitation energies, damping factors, and microscopic cross sections obtained from the deconvolution are collected in Table VI.

For trans-stilbene, the fits reveal at least two contributions to the broad 2PA band centered near $5.1 \mathrm{eV}$ : a weak band with excitation energy of $5.01 \mathrm{eV}$ (microscopic cross section of 702 a.u.) and a much stronger band centered near $5.27 \mathrm{eV}$ (5569 a.u.). The third band in the fit accounts for the large increase of the cross section above $\sim 5.6 \mathrm{eV}$. This higher-energy band represents the feature that was previously reported to have a maximum near $6.4 \mathrm{eV}$, but may also include contributions from the tails of other states at higher energy.

For comparison with the experimental deconvolution, Table II presents the calculated 2PA cross sections of transstilbene for parallel linearly polarized excitation and a pump wavelength of $380 \mathrm{~nm}$. We report both the microscopic and macroscopic cross sections. The two low-energy bands from the fit most closely match the $3{ }^{1} \mathrm{~A}_{g}$ and $4{ }^{1} \mathrm{~A}_{g}$ states with $\left\langle\delta_{\|}^{2 P A}\right\rangle$ of 1182 and 4070 a.u., respectively. Thus, Band 1 can be assigned as the transition to the $3{ }^{1} \mathrm{~A}_{g}$ state and Band 2 to the $4^{1} \mathrm{~A}_{g}$ state. With this assignment, the computed excitation energies are blue-shifted by $0.73 \mathrm{eV}$ and $0.88 \mathrm{eV}$ relative to the experiment. This difference is of the same magnitude as the difference in the 1PA spectra. Notably, this simple deconvolution

TABLE VI. Extrapolated experimental excitation energies, broadenings, and microscopic parallel cross sections of trans-stilbene, cis-stilbene, and phenanthrene.

\begin{tabular}{lcccc}
\hline \hline & $\omega_{i}(\mathrm{eV})$ & $\Gamma_{i}(\mathrm{eV})$ & $\left\langle\delta_{i}^{2 P A}\right\rangle$ (a.u.) & Tentative assignment \\
\hline trans-stilbene & & & & \\
\hline Band 1 & 5.01 & 0.26 & 702 & $3^{1} \mathrm{~A}_{g}$ \\
Band 2 & 5.27 & 0.63 & 5569 & $4^{1} \mathrm{~A}_{g}$ \\
Band 3 & 6.17 & 0.24 & 8652 & $\left(5^{1} \mathrm{~A}_{g}, \ldots\right)$ \\
\hline cis-stilbene & & & & \\
\hline Band 1 & 4.47 & 0.21 & 113 & $2^{1} \mathrm{~A}$ \\
Band 2 & 5.19 & 0.35 & 957 & $5^{1} \mathrm{~A}$ \\
Band 3 & 6.57 & 0.74 & 5514 & $\left(6^{1} \mathrm{~A}, \ldots\right)$ \\
\hline Phenanthrene & & & & \\
\hline Band 1 & 3.76 & 0.28 & 45 & $2^{1} \mathrm{~A}_{1}$ \\
Band 2 & 4.82 & 0.25 & 331 & $3^{1} \mathrm{~A}_{1}$ \\
Band 3 & 5.73 & 0.57 & 1930 & $\ldots$ \\
\hline \hline
\end{tabular}

does not recover the low-energy shoulder on the experimental spectrum near $4.3 \mathrm{eV}$, which can be assigned as the $2^{1} \mathrm{~A}_{g}$ state, based on the lower relative intensity of the calculated 2PA cross section and a shift of $\sim 0.5 \mathrm{eV}$. The higher-energy band in the fits may have significant contributions from the intense transition to $5^{1} \mathrm{~A}_{g}$. We note that the cross section from the fit is lower than the calculated value, and that the calculated energy is close to the experimentally observed band maximum even without corrections. These important differences may be related to the significant Rydberg character of the calculated $5^{1} \mathrm{~A}_{g}$ state, as discussed below.

The fit to the experimental spectrum of cis-stilbene using three Gaussian bands is more robust because of the more pronounced shoulder near $4.4 \mathrm{eV}$, in addition to the band near $5.2 \mathrm{eV}$ and the tail from higher-lying transitions. For the two lower-energy bands, we obtain microscopic cross sections of 113 and 957 a.u. and excitation energies of 4.47 and $5.19 \mathrm{eV}$, respectively. Based on a comparison with the calculated excitation energies and 2PA cross sections in Table III, we assign the first band as the $2^{1} \mathrm{~A}$ state (cross section of 171 a.u.) and the second band as the $5^{1} \mathrm{~A}$ state (1169 a.u.). Similar to trans-stilbene, the calculated cross sections are of the same order of magnitude as the experimental values, but the excitation energies of the $2^{1} \mathrm{~A}$ and $5^{1} \mathrm{~A}$ states are overestimated by $0.54 \mathrm{eV}$ and $1.00 \mathrm{eV}$, respectively. (Alternatively, Band 2 could be assigned as a combination of several weaker, overlapping transitions to $3^{1} \mathrm{~A}$, $4^{1} \mathrm{~A}$, and $4^{1} \mathrm{~B}$, with shifts of $0.6-0.8 \mathrm{eV}$, but the combined intensity of these transitions is lower than the experimental value from the fit.) As before, the higher-lying band may contain contributions from multiple states, possibly including the intense 2PA transition to $6^{1} \mathrm{~A}$. The large bandwidth of $0.74 \mathrm{eV}$ from the fit is consistent with this high-energy band having multiple contributions.

In phenanthrene, we observe a weak transition below $4 \mathrm{eV}$ and a pronounced shoulder near $4.8 \mathrm{eV}$. The lowest band seems to have some vibronic structure, although this is a difficult region of the spectrum to measure experimentally, due to overlapping Raman bands in the pump-probe measurement. ${ }^{82}$ Applying the simple three-Gaussian model gives an excitation energy of $3.76 \mathrm{eV}$ with a microscopic cross section of 45 a.u. for the lowest band, and an excitation energy of $4.82 \mathrm{eV}$ with a microscopic cross section of 331 a.u. for the second band. By comparing with the calculated energies and cross sections (Table IV), we assign the lowest-energy band as $2^{1} \mathrm{~A}_{1}$, on the basis of the calculated transition energy of 4.16 $\mathrm{eV}$ and $\left\langle\delta_{\|}^{2 P A}\right\rangle$ of 29 a.u., which requires a shift of only $\sim 0.4$ $\mathrm{eV}$. Other transitions could also contribute to the low-energy region, including $1^{1} \mathrm{~B}_{1}$ and $2^{1} \mathrm{~B}_{1}$, but these states would have to shift by more than $1 \mathrm{eV}$, which is inconsistent with the observed shifts in the 1PA spectra. With the weaker transitions in mind, we assign Band 2 as a collection of transitions with different symmetries, but dominated by the $3^{1} \mathrm{~A}_{1}$ state with a calculated excitation energy of $5.55 \mathrm{eV}$ and a microscopic cross section of 320 a.u. Other likely contributions include $2{ }^{1} \mathrm{~B}_{1}, 1^{1} \mathrm{~A}_{2}$, and $2^{1} \mathrm{~B}_{2}$, which are shifted by $0.3-0.9 \mathrm{eV}$ from the experimental band maximum. The fit to the higher-lying band in the 2PA spectrum may suffer from the drop in the experimental spectrum above $5.6 \mathrm{eV}$, but is likely to include contributions from several states, as evidenced by the broad linewidth parameter. 
Similar to the other compounds, our overall conclusion from the deconvolution of the 2PA spectrum of phenanthrene is that the computed cross sections are of the same magnitude as the values obtained from the fits, but the excitation energies are consistently overestimated by $0.4-0.7 \mathrm{eV}$.

\section{Analysis of the excited states giving rise to main features in the experimental spectra}

Analyzing the character of electronic states that are responsible for the main features in the experimental 2PA spectrum of each molecule provides valuable insight. Here we focus on the experimentally accessible states with transition energies below $\sim 6.5 \mathrm{eV}$; the higher-lying states from the calculation are discussed in Ref. 65. Several key properties ${ }^{61-63}$ of the excited-state wave functions are provided in Table VII, including average sizes of electron hole and a particle $\left(\sigma_{e}\right.$ and $\sigma_{h}$, their difference and a related quantity, $\Delta\left\langle R^{2}\right\rangle$, which represents the change in size of the wave function following excitation. ${ }^{65}$ We also report NTO participation ratio $\left(P R_{N T O}\right)$, which shows how many NTO pairs are needed to describe the transition, ${ }^{62,63}$ as well as two related quantities characterizing the one-electron character of the transition, the norm of one-particle transition density matrix $(\|\gamma\|)$, and the norm on single-excited EOM amplitudes $\left(\left\|R_{1}\right\|^{2}\right)$.

In general, all of the transitions in this low-energy region of the spectrum have relatively small $\Delta\left\langle R^{2}\right\rangle$, indicating that these states are either purely valence or mixed valence/Rydberg in character for all three molecules. The fate of Rydberg states in solution is unclear. It is possible that the states with a mixed character become more valence-like (i.e., less diffuse) upon solvation. In addition to the relative size of the wave function in the excited state, the NTOs associated with each transition are helpful in determining the relative valence/Rydberg character of the calculated states, as discussed below. Another important observation is that all of the excited states with significant 2PA cross sections in this energy range are singly excited states, as indicated by the values of $\left\|R_{1}\right\| \approx 0.9$ (see Ref. 65).

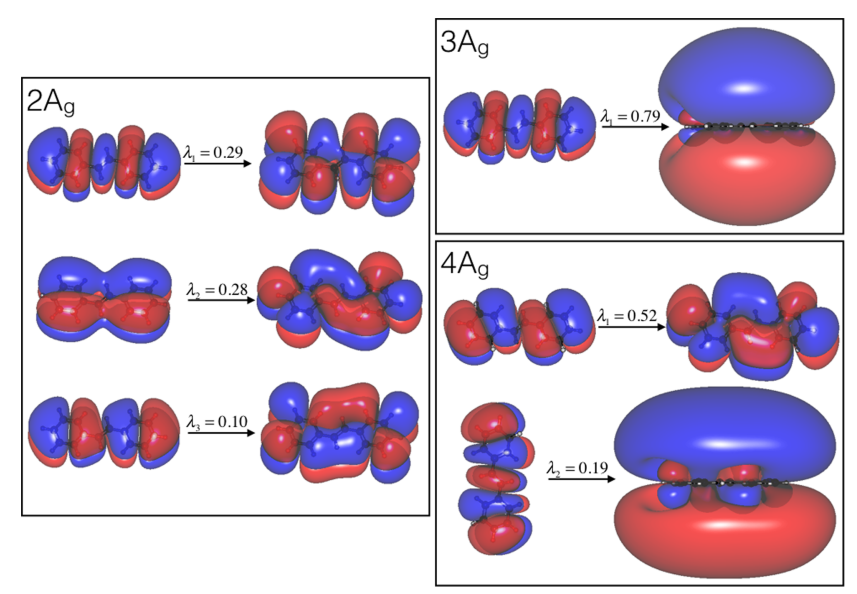

FIG. 6. NTOs corresponding to the $2{ }^{1} \mathrm{~A}_{g}, 3{ }^{1} \mathrm{~A}_{g}$, and $4{ }^{1} \mathrm{~A}_{g}$ excited states in trans-stilbene. The isovalue of $\sqrt{3 \cdot 10^{-6}}$ is used.

In the case of trans-stilbene, the assigned states from the fits include $2^{1} \mathrm{~A}_{g}, 3^{1} \mathrm{~A}_{g}$, and $4^{1} \mathrm{~A}_{g}$. Figure 6 shows the NTOs for each of these transitions. The $2^{1} \mathrm{~A}_{g}$ state has the smaller 2PA cross section of the three and consists of three $\pi \rightarrow \pi^{*}$ like excitations. This lowest-energy state has a pure valence character, as indicated by the small $\Delta\left\langle R^{2}\right\rangle$ of $0.1 \AA^{2}$. The $3^{1} \mathrm{~A}_{g}$ state can be described as $\pi \rightarrow p$ Rydberg transition $\left(\Delta\left\langle R^{2}\right\rangle\right.$ $=12.8 \AA^{2}$ ). We note that without the inclusion of this state in the simulated spectrum, it is impossible to reproduce the experimental shape, although it is the only state with a predominantly Rydberg character in the calculations that significantly contributes to the low-energy region of the experimental spectra. The $4^{1} \mathrm{~A}_{g}$ state has two pairs of NTOs (participation ratio of 2.06); the first (with $\lambda_{1}=0.52$ ) can be described as a 1PAforbidden $\pi \rightarrow \pi^{*}$ transition, and the second $\left(\lambda_{2}=0.19\right)$ as a $\pi \rightarrow p$ Rydberg transition. Despite some Rydberg contribution, this state is not very diffuse $\left(\Delta\left\langle R^{2}\right\rangle=2.2 \AA^{2}\right)$, indicating primarily a valence character.

The experimental 2PA spectrum of cis-stilbene includes additional broadening due to the non-planar structure of the molecule. Not only is the molecule more flexible, but also

TABLE VII. Key properties of the brightest 2PA states in trans-stilbene, cis-stilbene, and phenanthrene.

\begin{tabular}{lccccccccc}
\hline \hline State & $\left\|R_{1}\right\|^{2}$ & $\|\gamma\|$ & $\mathrm{PR}_{N T O}$ & $\left|\vec{d}_{h \rightarrow e}\right|, \AA$ & $\sigma_{h}, \AA$ & $\sigma_{e}, \AA$ & $R_{e h}$ & $\sigma_{e}-\sigma_{h}, \AA$ & $\Delta\left\langle R^{2}\right\rangle, \AA^{2}$ \\
\hline trans-stilbene & & & & & & & & & \\
\hline $2^{1} \mathrm{~A}_{g}$ & 0.893 & 0.872 & 3.27 & 0.0 & 3.65 & 3.76 & 0.62 & 0.11 & 0.1 \\
$3^{1} \mathrm{~A}_{g}$ & 0.930 & 0.913 & 1.11 & 0.0 & 3.07 & 5.16 & 0.13 & 2.09 & 12.8 \\
$4^{1} \mathrm{~A}_{g}$ & 0.918 & 0.903 & 2.06 & 0.0 & 3.61 & 4.03 & 0.39 & 0.42 & 2.2 \\
$5^{1} \mathrm{~A}_{g}$ & 0.879 & 0.856 & 1.75 & 0.0 & 3.08 & 4.80 & -0.05 & 1.72 & 10.2 \\
\hline cis-stilbene & & & & & & & & & \\
\hline $2^{1} \mathrm{~A}$ & 0.930 & 0.875 & 3.65 & 0.0 & 3.14 & 3.27 & 0.58 & 0.13 & 3.6 \\
$5^{1} \mathrm{~A}$ & 0.921 & 0.906 & 2.16 & 0.1 & 3.08 & 4.02 & 0.30 & 0.94 & 4.9 \\
$6^{1} \mathrm{~A}$ & 0.910 & 0.888 & 1.60 & 1.1 & 2.87 & 5.55 & 0.15 & 2.68 & 18.4 \\
\hline Phenanthrene & & & & & & & & & \\
\hline $2^{1} \mathrm{~A}_{1}$ & 0.892 & 0.873 & 2.21 & 0.1 & 2.75 & 2.88 & 0.23 & 0.13 & 0.0 \\
$3^{1} \mathrm{~A}_{1}$ & 0.905 & 0.886 & 1.89 & 0.4 & 2.70 & 3.27 & 0.06 & 0.57 & 1.9 \\
$2^{1} \mathrm{~B}_{1}$ & 0.900 & 0.879 & 3.11 & 0.2 & 2.74 & 3.03 & 0.35 & 0.29 & 0.5 \\
\hline \hline
\end{tabular}


the transition energies and intensities depend on the relative orientation of the two phenyl groups, leading to broader transitions relative to the other two molecules. Figure 7 shows the NTOs associated with the transitions to the $2^{1} \mathrm{~A}$ and $5^{1} \mathrm{~A}$ states of cis-stilbene. The former is a combination of three $\pi \rightarrow \pi^{*}$-like excitations, similar to the $2^{1} \mathrm{~A}_{g}$ state of transstilbene, and has a predominantly valence character $\left(\Delta\left\langle R^{2}\right\rangle\right.$ $=3.6 \AA^{2}$ ). This 2PA transition is weaker in the cis form of the molecule because of the non-planar structure, which inhibits $\pi$ conjugation between the phenyl rings and reduces exciton delocalization. ${ }^{65}$ The higher-lying $5^{1} \mathrm{~A}$ state is described by two pairs of NTOs; the leading one has a valence character and the second is a more diffuse $\pi \rightarrow p$-like transition. The mixed valence/Rydberg character is evident from the intermediate size of the excited-state wave function, $\Delta\left\langle R^{2}\right\rangle=4.9 \AA^{2}$.

The low-energy part of spectrum of phenanthrene is dominated by the three excited states $\left(2^{1} \mathrm{~A}_{1}, 2^{1} \mathrm{~B}_{1}\right.$, and $\left.3^{1} \mathrm{~A}_{1}\right)$. Except for the $2^{1} \mathrm{~A}_{1}$ state, all of these states are also active (but weak) in 1PA. The valence $2^{1} \mathrm{~A}_{1}$ state (Fig. 8) is the result of two $\pi \rightarrow \pi^{*}$ excitations. This state is similar to the $2^{1} \mathrm{~A}_{g}$ state of trans-stilbene and the $2^{1} \mathrm{~A}$ state of cis-stilbene. All three states have a predominantly valence character, as indicated by the small $\Delta\left\langle R^{2}\right\rangle$ in Table VII.

\section{Simulation of 2PA spectra}

A more complete simulation of the 2PA spectra should include all calculated transitions. However, such a simulation is complicated by the state-specific shifting and broadening of the transitions. As in the case of the 1PA spectra, several factors contribute to the discrepancy between the calculated excitation energies and the locations of the 2PA bands in the experimental spectra. Although we cannot systematically include all missing effects in the calculations, we can use the calculated transition energies and intensities and the results from the deconvolution of the experimental results above to simulate the 2PA spectra. These simulations allow us to evaluate the degree of shifting and broadening that is required to bring the theoretical spectra into agreement with the experimental spectra on a state-bystate level.

While we do not have a reliable way to predict a priori the effect of higher-order excitations and solvatochromic shifts in these relatively large molecules, we can evaluate possible vibronic effects using the estimated adiabatic energies (computed using cationic geometries, as described in Section III B). Adiabatic excitation energies for selected states are reported

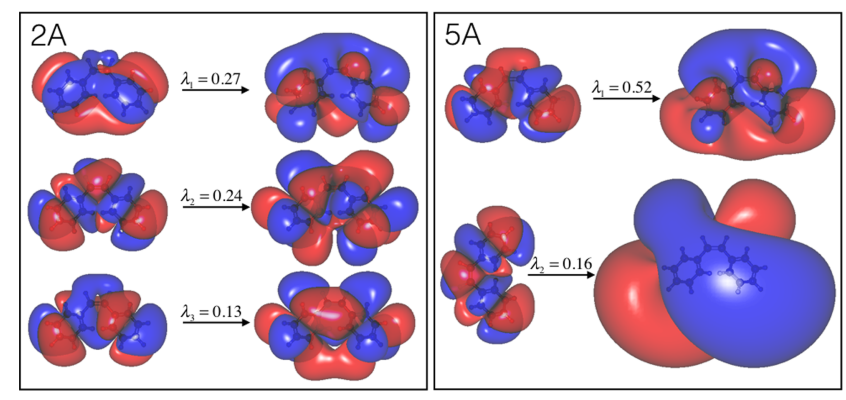

FIG. 7. NTOs corresponding to the $2^{1} \mathrm{~A}$ and $5^{1} \mathrm{~A}$ excited states in cis-stilbene. The isovalue of $\sqrt{3 \cdot 10^{-6}}$ is used.

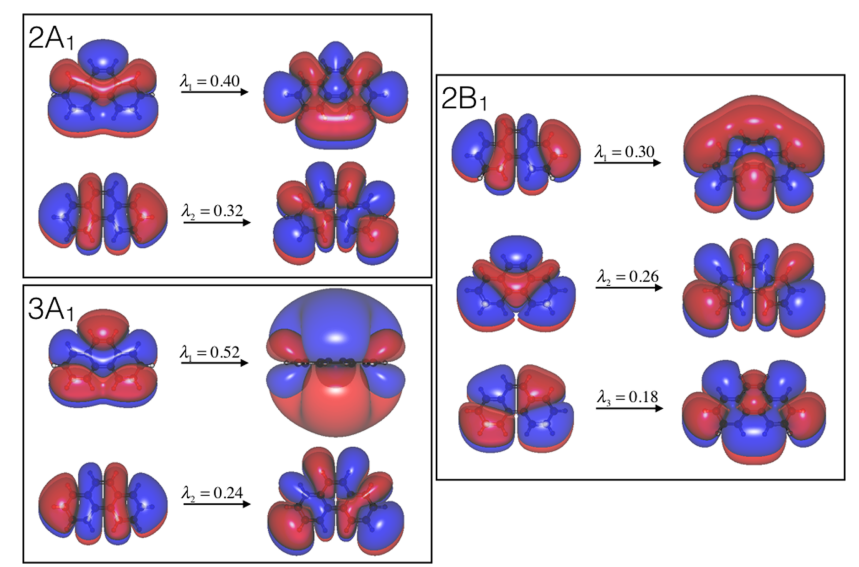

FIG. 8. NTOs corresponding to the $2{ }^{1} \mathrm{~A}_{1}, 3{ }^{1} \mathrm{~A}_{1}$, and $2{ }^{1} \mathrm{~B}_{1}$ excited states in phenanthrene. The isovalue of $\sqrt{3 \cdot 10^{-6}}$ is used.

in Table $\mathrm{S} 1$ of the supplementary material. The calculated adiabatic energies suggest that vibronic effects could result in red shifts of up to $0.2 \mathrm{eV}$ for the $3^{1} \mathrm{~A}_{g}$ and $4^{1} \mathrm{~A}_{g}$ states of transstilbene. For cis-stilbene, geometric relaxation can lower the excitation energy of the $5^{1} \mathrm{~A}$ state by as much as $0.26 \mathrm{eV}$, but has no effect on the $2^{1} \mathrm{~A}$ state. And in phenanthrene, we observe red shifts of 0.05 and $0.12 \mathrm{eV}$ for the $2^{1} \mathrm{~A}_{1}$ and $3^{1} \mathrm{~A}_{1}$ states, respectively. For trans-stilbene, we also examined basis-set effects by comparing the adiabatic excitation energies obtained with the d-aug-cc-pVDZ and d-aug(-df)-cc-pVTZ basis sets. Changing the basis set appears to have only a minimal impact. Adding the missing diffuse $d$ functions in the doubly augmented basis affects only the $3^{1} \mathrm{~A}_{g}$ state, for which the energy increases by $0.03 \mathrm{eV}$ relative to the $\mathrm{d}-\mathrm{aug}(-\mathrm{d})-\mathrm{cc}-\mathrm{pVDZ}$ basis set. Going from a double- $\zeta$ to a triple- $\zeta$ basis set results in $0.1 \mathrm{eV}$ blue shift for the $3^{1} \mathrm{~A}_{g}$ state. In sum, taking into account vibronic structure of the bands may lead to red shifts of up to $0.2 \mathrm{eV}$. Together with typical error bars of $0.3 \mathrm{eV}$ for EOM-CCSD, this could account for up to $\sim 0.5 \mathrm{eV}$ discrepancy between the theoretical and experimental excitation energies, similar to the 1PA transitions. Solvation can cause additional shifts, as discussed in Section III B.

Using these considerations, we apply a systematic approach to simulate the 2PA spectra, with some modifications based on the fits to the experimental spectra. Fig. 9 compares the simulated and experimental spectra; specific details for each molecule are given below. We use the adiabatically corrected excitation energies (see Table $\mathrm{S} 4$ of the supplementary material) and then apply an additional red shift of $0.40 \mathrm{eV}$ to the states with low excitation energies. We shift only the lower transition energies, because of smaller solvatochromic shifts that we observed for higher-energy states in the 1PA spectrum of trans-stilbene and because the more diffuse states at higher energy are expected to blue-shift due to confinement effects of the solvent. Although the fate of Rydberg states upon solvation remains an open question, ${ }^{87}$ it is likely that more diffuse states will experience a blue shift due to Pauli repulsion that partially compensates the errors due to approximate correlation treatment. Finally, we also apply a damping factor of $0.2 \mathrm{eV}$ to all of the excited states below $6.0 \mathrm{eV}$, except for the lowenergy states with the largest cross sections, for which we use 

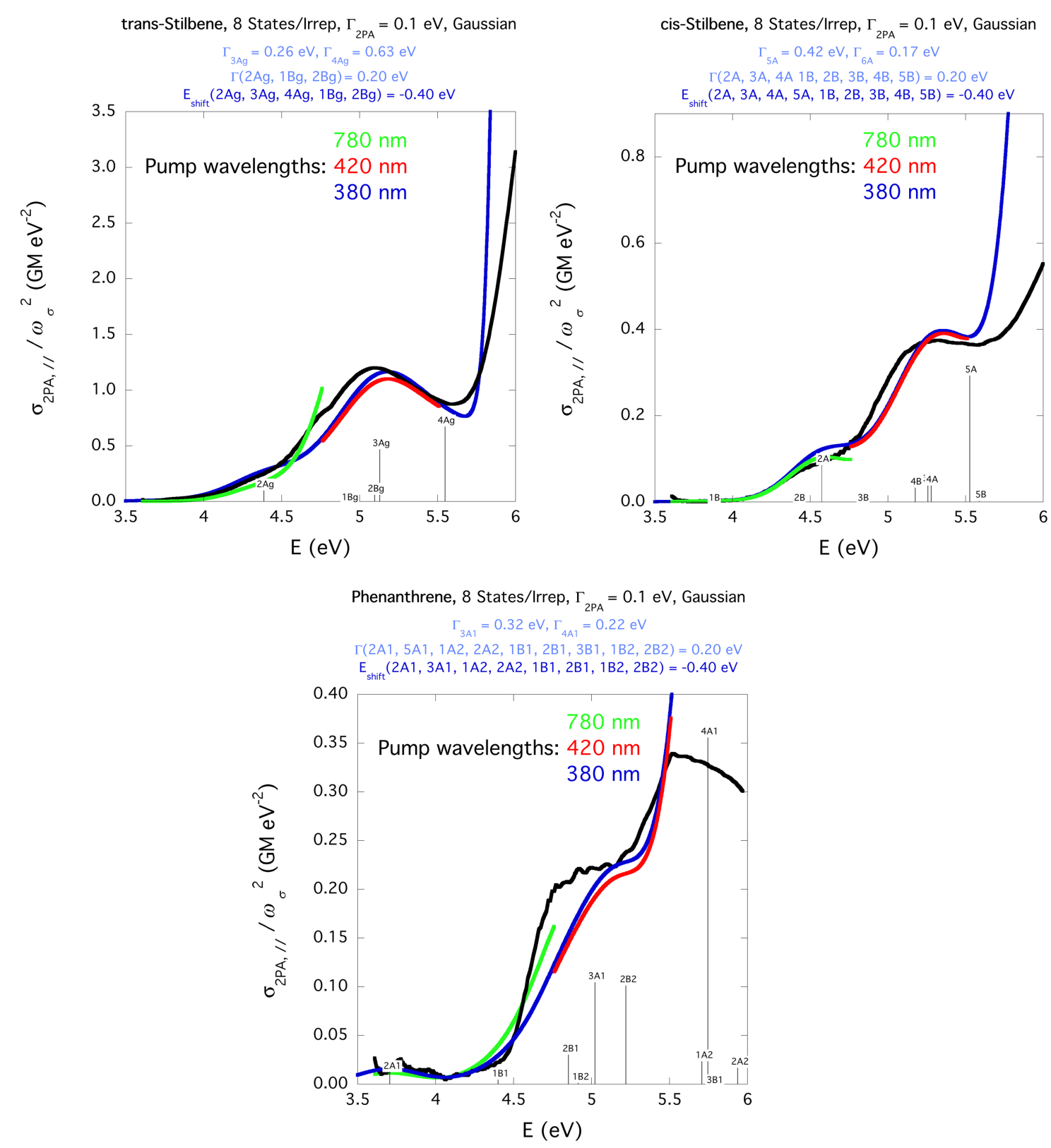

FIG. 9. Comparison between the experimental and theoretical (\|) 2PA spectra of trans-stilbene, cis-stilbene, and phenanthrene. The damping factors of the theoretical spectra have been chosen on the basis of the fitting of the experimental spectra.

damping factors extracted from the fitting of experimental spectra in Sec. III C 3. For the excited states above $6.0 \mathrm{eV}$, we use a damping factor of $0.1 \mathrm{eV}$, to prevent the low-energy tails of those transitions from spilling into the lower-energy region of the spectrum (and to account for the reduced contribution from Rydberg states in solution).

The so-simulated spectrum of trans-stilbene reproduces reasonably well the main features of the experimental $2 \mathrm{PA}$ spectrum. The spectrum includes damping factors of $0.26 \mathrm{eV}$ and $0.63 \mathrm{eV}$ for the $3^{1} \mathrm{~A}_{g}$ and $4^{1} \mathrm{~A}_{g}$ states that were extracted from the fit to the experimental spectrum. Note that the $5^{1} \mathrm{~A}_{g}$ state, which has the largest calculated 2PA cross section (and significant Rydberg character), was not shifted to lower energy. This simulation confirms the primary role of the transitions to $2^{1} \mathrm{~A}_{g}, 3^{1} \mathrm{~A}_{g}$, and $4^{1} \mathrm{~A}_{g}$ in the lower-energy region of the 2PA spectrum of trans-stilbene, with minor contributions from weaker overlapping transitions. As suggested above, the simulation also predicts that the strong 2PA band, previously reported to have a maximum near $6.4 \mathrm{eV}$, may result from a transition to the $5^{1} \mathrm{~A}_{g}$ state, but with lower intensity and little or no energy shift relative to the calculation.

For $c i s$-stilbene, we use damping factors of $0.20 \mathrm{eV}$ for the $2^{1} \mathrm{~A}$ state and $0.42 \mathrm{eV}$ for the $5^{1} \mathrm{~A}$ state. The damping factor of $0.42 \mathrm{eV}$ is slightly larger than the value of $0.35 \mathrm{eV}$ obtained from fitting the experimental spectrum in order to compensate for the larger 2PA cross section, and a broadening factor of only $0.17 \mathrm{eV}$ was used for the $6^{1} \mathrm{~A}$ state to better reproduce the shape of the spectrum. As before, the overestimate of the 2PA cross section at the highest energies points to a reduced contribution from Rydberg transitions in the experimental solution-phase spectra. Based on other adjustments, 

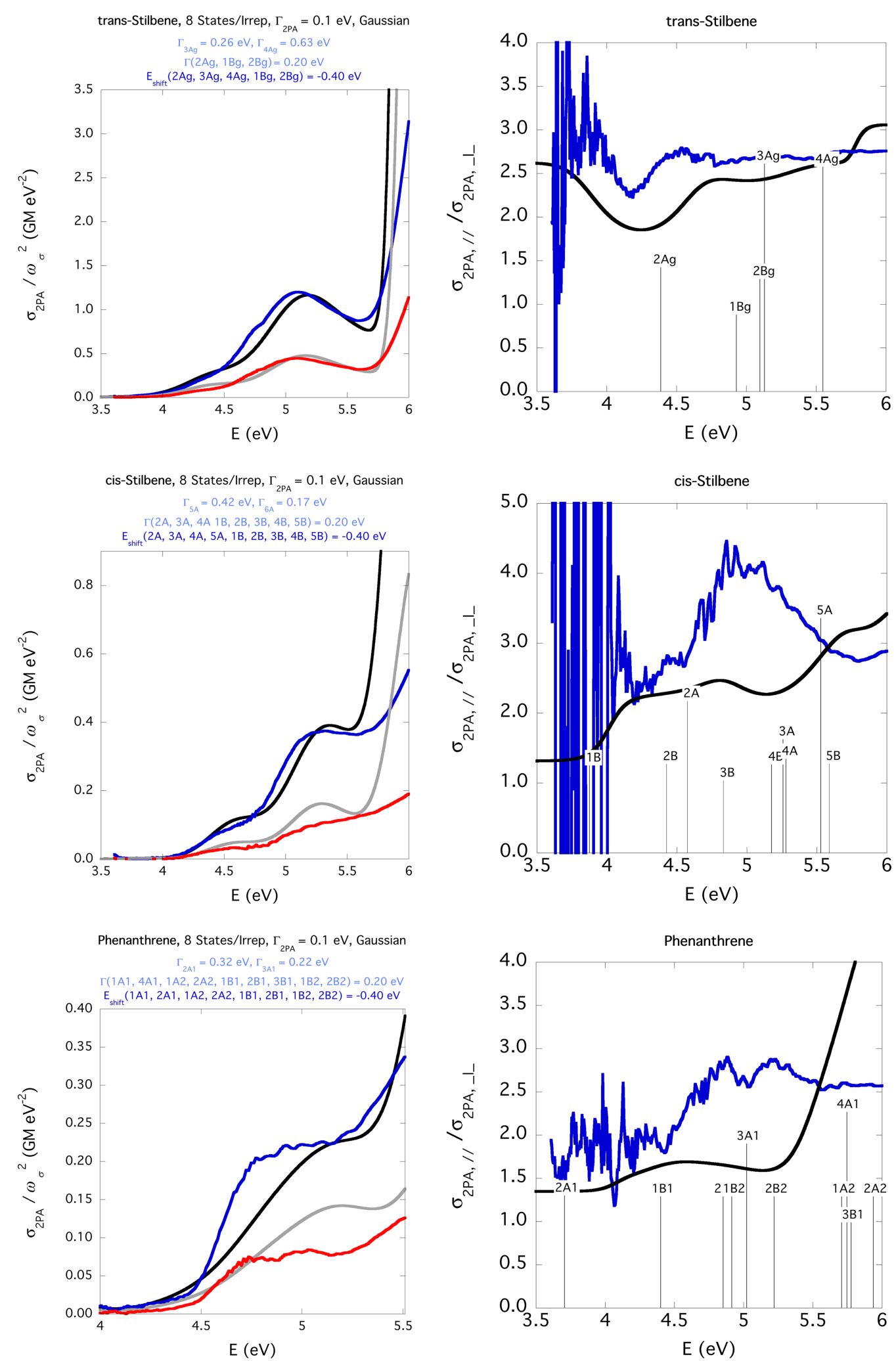

FIG. 10. Comparison between the experimental and theoretical $(\|[$ exp. $=$ blue, theo. $=$ black $]$ and $\perp[$ exp. $=$ red, theo. $=$ gray $]) 2 \mathrm{PA}$ spectra and polarization ratios of trans-stilbene, cis-stilbene, and phenanthrene. The damping factors used in the theoretical spectra were taken from the fitting of the experimental spectra.

however, we identify the excited states that define the shape of the 2PA spectrum. We also note that the contributions from a small cluster of states around $\sim 5.6 \mathrm{eV}\left(3^{1} \mathrm{~A}, 4^{1} \mathrm{~A}\right.$, and $\left.4^{1} \mathrm{~B}\right)$ are important for recovering the spectral shape between 5.0 and $5.5 \mathrm{eV}$.
Finally, for phenanthrene, we shift all states with adiabatic excitation energies below $5.5 \mathrm{eV}$ to lower energy by $0.4 \mathrm{eV}$, except for the $4^{1} \mathrm{~A}_{1}$ and $5^{1} \mathrm{~A}_{1}$ states, which have the largest cross sections. We also used a larger damping factor of $0.32 \mathrm{eV}$ for the $3^{1} \mathrm{~A}_{1}$ state, in order to compensate for the larger 
calculated cross section compared with the value obtained from the fit, and a damping factor of $0.22 \mathrm{eV}$ for the $4^{1} \mathrm{~A}_{1}$ state. As in the two other molecules, we used a damping factor of $0.2 \mathrm{eV}$ for all other states below $6.0 \mathrm{eV}$, including the $2^{1} \mathrm{~A}_{1}$ state, because the value obtained from the fit to that low-energy band is not sufficiently accurate due to the low intensity and vibronic structure.

Although the agreement between the experimental and simulated 2PA spectra in Fig. 9 is not perfect, the fact that we are able to reproduce the general shapes of the experimental solution-phase spectra using a few simple manipulations of the calculated gas-phase results is quite encouraging. The remaining discrepancies highlight the need for further theoretical work to better understand the role of electron correlation, vibronic structure, and, especially, interaction with the solvent. Together, these effects result in shifts of $\sim 0.4-0.7 \mathrm{eV}$ for the states in the low-energy region of the spectrum. In Sec. III C 5, we examine the polarization dependence of the 2PA spectra, to further validate our assignments.

\section{2PA polarization ratios}

The polarization dependence of the 2PA spectrum provides an experimental measure of the electronic symmetry of the underlying transitions. ${ }^{75,76,83}$ Here, we quantify the polarization dependence as the ratio of the 2PA cross section for parallel and perpendicular linearly polarized light, $r$ $=\sigma_{\|}^{2 P A} / \sigma_{\perp}^{2 P A}$. Based on the sum-over-states expression, the microscopic 2PA cross section of a single transition depends on the relative intensities of the individual components of the transition strength matrix, $S_{a b, c d} .{ }^{65}$ In the $C_{2 v}$ point group, for totally symmetric $\mathrm{A}_{1}$ excited states, the $2 \mathrm{PA}$ microscopic cross section reads ${ }^{65}$

$$
\left\langle\delta^{2 P A}\right\rangle_{n A_{1} \leftarrow 1 A_{1}}=\begin{gathered}
\frac{F}{30}\left(\begin{array}{c}
S_{x x, x x}+S_{x x, y y}+S_{x x, z z}+S_{y y, x x}+S_{y y, y y} \\
+
\end{array} S_{y y, z z}+S_{z z, x x}+S_{z z, y y}+S_{z z, z z}\right. \\
+ \\
+\frac{G}{30}\left(S_{x x, x x}+S_{y y, y y}+S_{z z, z z}\right) \\
+\frac{H}{30}\left(S_{x x, x x}+S_{y y, y y}+S_{z z, z z}\right),
\end{gathered}
$$

where $F=G=H=2$ for parallel and $F=-1, G=4, H=-1$ for perpendicular linear polarization. ${ }^{75}$ Thus, if the off-diagonal components of the transition strength matrix $S_{i i, j j}$ are small relative to the diagonal terms, $S_{i i, i i}$, then one obtains the limiting value $r=\frac{\left\langle\delta_{\|}^{2 P A}\right\rangle}{\left\langle\delta_{\perp}^{2 P A}\right\rangle}=3$. In any case, the ratio is restricted to $r>4 / 3$ for the totally symmetric irreducible representation. For other irreducible representations, e.g., $\mathrm{A}_{2}$, the two-photon transition dipole tensor contains non-zero off-diagonal elements, and the microscopic cross section becomes

$$
\left\langle\delta^{2 P A}\right\rangle_{n A_{2} \leftarrow 1 A_{1}}=\frac{G}{30}\left(S_{x y, x y}+S_{y x, y x}\right)+\frac{H}{30}\left(S_{x y, y x}+S_{y x, x y}\right),
$$

with $G$ and $H$ as before. In the degenerate case, $M_{x y}^{k \leftarrow 0}=M_{y x}^{k \leftarrow 0}$ and $M_{x y}^{k \rightarrow 0}=M_{y x}^{k \rightarrow 0}: r=\frac{\left\langle\delta_{\|}^{2 P A}\right\rangle}{\left\langle\delta_{\perp}^{2 P A}\right\rangle}=\frac{4}{3}$. In the non-degenerate case $\left(\omega_{1} \neq \omega_{2}\right)$, the ratio is slightly different, but always $\leq 4 / 3$. These symmetry-imposed rules for totally symmetric and not totally symmetric transitions are also valid for $C_{2 h}$ and $C_{2}$ point groups.
Fig. 10 compares the experimental and theoretical 2PA spectra and polarization ratios of trans-stilbene, cis-stilbene, and phenanthrene. We use the same damping factors for the parallel and perpendicular 2PA spectra because the transitions access the same final state in each case, and only the relative intensity of the transition (not the width) depends on the relative polarization of the pump and probe light. For transstilbene, the 2PA polarization ratio confirms the symmetry assignment of the $2^{1} \mathrm{~A}_{g}, 3^{1} \mathrm{~A}_{g}$, and $4^{1} \mathrm{~A}_{g}$ excited states. The $3^{1} \mathrm{~A}_{g}$ and $4^{1} \mathrm{~A}_{g}$ ratios are close to 3 , but the ratio is smaller for $2^{1} \mathrm{~A}_{g}$ due to larger contributions from the off-diagonal elements of the two-photon transition strength matrix.

For cis-stilbene, the agreement between the experimental ratio and the computed one (using the same damping factors for parallel and perpendicular spectra) is not as good as in trans-stilbene, because the experimental ratio increases around $\sim 5 \mathrm{eV}$ due to the flatter $2 \mathrm{PA}$ spectrum for perpendicular polarization in the region of 5-5.5eV. The $2^{1} \mathrm{~A}$ and $5^{1} \mathrm{~A}$ states, which dominate the simulated spectrum in this region, have ratios of 2.2 and 3.4, respectively, whereas the weaker, underlying $3^{1} \mathrm{~A}$ and $4^{1} \mathrm{~A}$ states have much lower ratios. Interestingly, the calculated polarization ratio for the $7^{1} \mathrm{~A}$ state $\left(\Delta\left\langle R^{2}\right\rangle=20.0 \AA^{2}\right)$ is $r=8.5$, which could explain the higher polarization ratio, but would require a shift of $>1 \mathrm{eV}$ from the calculated transition energy of $6.34 \mathrm{eV}$.

For phenanthrene, as for cis-stilbene, the perpendicular experimental spectrum is mostly flat across the entire range. The ratios for the $2^{1} \mathrm{~A}_{1}$ and $3^{1} \mathrm{~A}_{1}$ transitions are 1.4 and 1.8 , respectively, due to non-negligible off-diagonal elements $\left(S_{i i, j j}\right)$. Consistent with the calculated ratios, phenanthrene has the lowest average polarization ratio of all three compounds. Overall, $r$ is slightly below 3 .

Although the agreement with experiment is not perfect, the simulated polarization ratios highlight the value of the polarization dependence as a means of separating the relative contributions from broad, overlapping transitions. Additionally, symmetry information is not generally available from 1PA spectra of disordered samples, other than parity selection rules, due to orientational averaging. Finally, we note that the polarization ratio is very sensitive to experimental conditions; therefore, theoretical ratios are important for interpreting the experimental spectra.

\section{CONCLUSION}

In this paper, we have analyzed the 2PA spectra of transstilbene, cis-stilbene, and phenanthrene, by comparing our experimentally measured broadband 2PA spectra with the theoretical spectra computed using EOM-EE-CCSD wave functions. The experimental spectra provide important benchmarks for comparison with the theoretical results, including a detailed simulation that blends information from both the experimental and theoretical spectra in order to better understand both. The experimental spectra themselves are unique in that we have measured the polarization-dependent broadband spectra for all compounds under identical conditions for the first time, and at a level of unprecedented resolution that allows us to comment on several fundamental features that are important for the comparison with the calculated 2PA spectra. 
The long-standing role of stilbene as a model compound makes it an ideal target for our experimental-theoretical comparison. Although the 2PA spectrum of stilbene has been subject of experimental studies since the 1970s, here we report 2PA spectra of both cis- and trans-isomers, as well as the closely related phenanthrene, under identical conditions and with unprecedented resolution. The comparison across this series of compounds reveals new details about the fundamental features of the 2PA spectra, and, more generally, the non-linear properties of these molecules. Our broadband measurements give accurate, absolute 2PA cross sections, whereas previously reported cross sections vary by orders of magnitude ${ }^{66}$ and have never been measured consistently for the set of all three compounds. Additionally, our broadband 2PA technique provides continuous 2PA spectra across a wide range of energies, which allows us to determine that there is no significant vibronic structure in the non-linear spectra, in contrast with the linear absorption spectra. This point was previously mis-interpreted based on the artifactual structure in the earlier experimental spectra that were obtained on a point-by-point basis using a single-wavelength technique. ${ }^{33}$ Vibronic structure is a key sticking point in calculating molecular spectra; therefore, this point of comparison is absolutely critical for developing predictive theoretical approaches. Earlier spectra also did not resolve subtle features that are important for comparison with the calculated spectra. In addition, the polarization dependence for linearly polarized light has not been reported before now for cis-stilbene or phenanthrene, yet that measurement provides another critical reference point for benchmarking the calculated spectra. Thus, our broadband spectra provide key insight into the fundamental details that must be considered when simulating solution-phase broadband 2PA spectra based on calculated gas-phase transition energies and intensities.

While the computed spectra reproduce the main features in the experimental spectra reasonably well, the quantitative comparison is complicated by several factors, including the non-uniform broadening and shifting of the experimental bands due to vibronic effects, solvation, etc. Combined with the inherent limitations of the computational method, the calculated transition energies typically are overestimated by as much as $0.4-0.7 \mathrm{eV}$. The frequency shifts of the calculated transition energies are exacerbated in the simulated broadband spectra due to an $\omega_{\sigma}^{2}$ dependence when calculating the macroscopic 2PA cross section. Additionally, we emphasize that the 2PA intensities implicitly depend on both the energies and the intensities of the intermediate 1PA transitions (by virtue of the sum-over-states expression); therefore, any errors in the 1PA transitions are amplified when calculating 2PA spectra.

The main stumbling block for comparing the calculated gas-phase spectra with the condensed-phase spectra is the uncertain fate of Rydberg states in solution. The most intense spectral features in the calculations correspond to Rydberg states, which can be rationalized by analyzing the sum-overstates expression for calculating microscopic 2PA cross sections. ${ }^{65}$ The fate of Rydberg states upon solvation is still a matter of debate, and the predictive power of theory is limited by the inability to reliably compute solvent shifts. Calculations that are capable of describing solvatochromic shifts of Rydberg states, such as effective fragment potential method, ${ }^{94-96}$ may be able help to clarify this issue with the help of experimental benchmarks. Nevertheless, the gas-phase calculations provide a reasonably good representation of the experimental 2PA spectra based on phenomenological broadening and shifts to lower energy by up to $0.4-0.7 \mathrm{eV}$ compared with the calculated transition energies. These results provide promising evidence that the experimental 2PA spectra can be interpreted with the help of ab initio calculations and careful wave function analysis.

\section{SUPPLEMENTARY MATERIAL}

See supplementary material for additional details and relevant Cartesian geometries.

\section{ACKNOWLEDGMENTS}

A.I.K. acknowledges support by the U.S. National Science Foundation (Grant No. CHE-1264018). C.G.E. acknowledges support from the U.S. National Science Foundation through a CAREER Award (No. CHE-1151555). We are grateful to Dr. Kaushik Nanda for valuable discussions and his help with the calculations. We thank Dr. Thomas Jagau for his feedback about the manuscript.

${ }^{1}$ W. R. Zipfel, R. M. Williams, and W. W. Webb, "Nonlinear magic: Multiphoton microscopy in the biosciences," Nat. Biotechnol. 21, 1369 (2003).

${ }^{2}$ F. Helmchen and W. Denk, "Deep tissue two-photon microscopy," Nat. Methods 2, 932 (2005).

${ }^{3}$ K. Konig, "Multiphoton microscopy in life sciences," J. Microsc. 200, 83 (2000).

${ }^{4}$ A. Diaspro, G. Chirico, and M. Collini, "Two-photon fluorescence excitation and related techniques in biological microscopy," Q. Rev. Biophys. 38, 97 (2006).

${ }^{5}$ A. Hayat, A. Nevet, P. Ginzburg, and M. Orenstein, "Applications of two-photon processes in semiconductor photonic devices," Semicond. Sci. Technol. 26, 083001 (2011).

${ }^{6}$ M. Pawlicki, H. A. Collins, R. G. Denning, and H. L. Anderson, "Twophoton absorption and the design of two-photon dyes," Angew. Chem., Int. Ed. 48, 3244 (2009).

${ }^{7}$ B. Strehmel and V. Strehmel, "Two-photon physical, organic, and polymer chemistry: Theory, techniques, chromophore design, and applications," in Advances in Photochemistry, edited by D. C. Neckers, W. S. Jenks, and T. Wolff (John Wiley \& Sons, Inc., Hoboken, NJ, USA, 2007), Vol. 29, pp. 111-351.

${ }^{8}$ G. S. He, L.-S. Tan, Q. Zheng, and P. N. Prasad, "Multiphoton absorbing materials: Molecular designs, characterizations, and applications," Chem. Rev. 108, 1245 (2008).

${ }^{9}$ H. M. Kim and B. R. Cho, "Two-photon materials with large two-photon cross sections. Structure property relationship," J. Chem. Soc., Chem. Commun. 2009, 153.

${ }^{10}$ C. Chung, D. Srikun, C. S. Lim, C. J. Chang, and B. R. Cho, "A twophoton fluorescent probe for ratiometric imaging of hydrogen peroxide in live tissue," J. Chem. Soc., Chem. Commun. 47, 9618 (2011).

${ }^{11}$ S. Yao and K. D. Belfield, "Two-photon fluorescent probes for bioimaging," Eur. J. Org. Chem. 2012, 3199.

${ }^{12} \mathrm{H}$. Yu, Y. Xiao, and L. Jin, "A lysosome-targetable and two-photon fluorescent probe for monitoring endogenous and exogenous nitric oxide in living cells," J. Am. Chem. Soc. 134, 17486 (2012).

${ }^{13}$ L. Li, J. Ge, H. Wu, Q.-H. Xu, and S. Q. Yao, "Organelle-specific detection of phosphatase activities with two-photon fluorogenic probes in cells and tissues," J. Am. Chem. Soc. 134, 12157 (2012).

${ }^{14}$ H.-Y. Ahn, K. E. Fairfull-Smith, B. J. Morrow, V. Lussini, B. Kim, M. V. Bondar, S. E. Bottle, and K. D. Belfield, "Two-photon fluorescence microscopy imaging of cellular oxidative stress using profluorescent nitroxides," J. Am. Chem. Soc. 134, 4721 (2012). 
${ }^{15}$ H. J. Kim, J. H. Han, M. K. Kim, C. S. Lim, H. M. Kim, and B. R. Cho, "Dualcolor imaging of sodium/calcium ion activities with twophoton fluorescent probes," Angew. Chem., Int. Ed. 49, 6786 (2010).

${ }^{16}$ T. Kowada, J. Kikuta, A. Kubo, M. Ishii, H. Maeda, S. Mizukami, and K. Kikuchi, "In vivo fluorescence imaging of bone-resorbing osteoclasts," J. Am. Chem. Soc. 133, 17772 (2011).

${ }^{17}$ L. Yuan, W. Lin, H. Chen, S. Zhu, and L. He, "A unique family of rigid analogues of the GFP chromophore with tunable two-photon action crosssections for biological imaging," Angew. Chem., Int. Ed. 52, 10018-10022 (2013).

${ }^{18}$ F. Terenziani, C. Katan, E. Badaeva, S. Tretiak, and M. Blanchard-Desce, "Enhanced two-photon absorption of organic chromophore: Theoretical and experimental assessments," Adv. Mater. 20, 4641 (2008).

${ }^{19}$ M. T. P. Beerepoot, D. H. Friese, and K. Ruud, "Intermolecular charge transfer enhances two-photon absorption in yellow fluorescent protein," Phys. Chem. Chem. Phys. 16, 5958 (2014).

${ }^{20}$ P. Salek, O. Vahtras, J. Guo, Y. Luo, T. Helgaker, and H. Årgen, "Calculations of two-photon absorption cross sections by means of density-functional theory," Chem. Phys. Lett. 374, 446 (2003).

${ }^{21}$ M. J. Paterson, O. Christiansen, F. Pawlowski, P. Jørgensen, C. Hättig, T. Helgaker, and P. Salek, "Benchmarking two-photon absorption with CC3 quadratic response theory, and comparison with density-functional response theory," J. Chem. Phys. 124, 054332 (2006).

${ }^{22}$ D. H. Friese, C. Hättig, and K. Ruud, "Calculation of two-photon absorption strengths with the approximate coupled cluster singles and doubles model CC2 using the resolution-of-identity approximation," Phys. Chem. Chem. Phys. 14, 1175 (2012).

${ }^{23}$ K. D. Nanda and A. I. Krylov, "Two-photon absorption cross sections within equation-of-motion coupled-cluster formalism using resolution-ofthe-identity and Cholesky decomposition representations: Theory, implementation, and benchmarks," J. Chem. Phys. 142, 064118 (2015).

${ }^{24}$ D. H. Waldeck, "Photoisomerization dynamics of stilbenes," Chem. Rev. 91, 415 (1991).

${ }^{25}$ S. T. Repinec, R. J. Sension, A. Z. Szarka, and R. M. Hochstrasser, "Femtosecond laser studies of the cis-stilbene photoisomerization reactions: The cis-stilbene to dihydrophenanthrene reaction,” J. Phys. Chem. 95, 10380 (1991).

${ }^{26} \mathrm{~J}$. Quenneville and T. J. Martinez, "Ab initio study of cis-trans photoisomerization in stilbene and ethylene," J. Phys. Chem. A 107, 829 (2003).

${ }^{27}$ A. Weigel and N. P. Ernsting, "Excited stilbene: Intramolecular vibrational redistribution and solvation studied by femtosecond stimulated raman spectroscopy," J. Phys. Chem. B 114, 7879 (2010).

${ }^{28} \mathrm{H}$. Meier, "The photochemistry of stilbenoid compounds and their role in materials technology," Angew. Chem., Int. Ed. 31, 1399 (1992).

${ }^{29}$ M. Irie, "Diarylethenes for memories and switches," Chem. Rev. 100, 1685 (2000).

${ }^{30}$ E. R. Kay, D. A. Leigh, and F. Zerbetto, "Synthetic molecular motors and mechanical machines," Angew. Chem., Int. Ed. 46, 72 (2007).

${ }^{31}$ M. F. Budyka, "Diarylethylene photoisomerization and photocyclization mechanisms," Russ. Chem. Rev. 81, 477 (2012).

${ }^{32}$ M. Irie, T. Fukaminato, K. Matsuda, and S. Kobatake, "Photochromism of diarylethene molecules and crystals: Memories, switches, and actuators," Chem. Rev. 114, 12174 (2014).

${ }^{33} \mathrm{G}$. Hohlneicher and B. Dick, "Experimental determination of the low-lying excited a states of trans-stilbene," J. Photochem. 27, 215 (1984).

${ }^{34}$ Z. G. Soos, S. Ramasesha, D. S. Galvao, and S. Etemad, "Excitation and relaxation energies of trans-stilbene: Confined singlet, triplet, and charged bipolarons," Phys. Rev. B 47, 1742 (1993).

${ }^{35}$ M. J. Bearpark, F. Bernardi, S. Clifford, M. Olivucci, M. A. Robb, and T. Vreven, "Cooperating rings in cis-stilbene lead to an $\mathrm{S}_{0} / \mathrm{S}_{1}$ conical intersection," J. Phys. Chem. A 101, 3841 (1997).

${ }^{36}$ S. P. Kwasniewski, M. S. Deleuze, and J. P. Francois, "Optical properties of trans-stilbene using semiempirical and time-dependent density functional theory: A comparative study," Int. J. Quant. Chem. 80, 672 (2000).

${ }^{37}$ C. W. M. Castleton and W. Barford, "Screening and the quantitative $\pi-$ model description of the optical spectra and polarizations of phenyl based oligomers," J. Chem. Phys. 117, 3570 (2000).

${ }^{38}$ P. C. Chen and Y. C. Chieh, "Azobenzene and stilbene: A computational study,” J. Mol. Struct.: THEOCHEM 624, 191 (2003).

${ }^{39}$ R. Improta and F. Santoro, "Excited-state behavior of trans and cis isomers of stilbene and stiff stilbene: A TD-DFT study," J. Phys. Chem. A 109, 10058 (2005).
${ }^{40}$ C. Angeli, R. Improta, and F. Santoro, "On the controversial nature of the $1^{1} \mathrm{~B}_{u}$ and $2^{1} \mathrm{~B}_{u}$ states of trans-stilbene: The n-electron valence state perturbation theory approach," J. Chem. Phys. 130, 174307 (2009).

${ }^{41}$ N. Minezawa and M. S. Gordon, "Photoisomerization of stilbene: A spin-flip density functional theory approach," J. Phys. Chem. A 115, 7901 (2011).

${ }^{42}$ R. K. Chaudhuri, K. F. Freed, S. Chattopadhyay, and U. S. Mahapatra, "Theoretical studies of the ground and excited state structures of stilbene," J. Phys. Chem. A 117, 9424 (2013).

${ }^{43}$ I. N. Ioffe and A. A. Granovsky, "Photoisomerization of stilbene: The detailed XMCQDPT2 treatment," J. Chem. Theory Comput. 9, 4973 (2013).

${ }^{44}$ A. K. Pal, S. Hansda, S. N. Datta, and F. Illas, "Theoretical investigation of stilbene as photochromic spin coupler," J. Phys. Chem. A 117, 1773 (2013).

${ }^{45}$ G. Tomasello, M. Garavelli, and G. Orlandi, "Tracking the stilbene photoisomerization in the $\mathrm{S}_{1}$ state using RASSCF," Phys. Chem. Chem. Phys. 15, 19763 (2013).

${ }^{46}$ Y. Harabuchi, K. Keipert, F. Zahariev, T. Taketsugu, and M. S. Gordon, "Dynamics simulations with spin-flip time-dependent density functional theory: Photoisomerization and photocyclization mechanisms of cisstilbene in $\pi \pi^{*}$ states," J. Phys. Chem. A 118, 11987 (2014).

${ }^{47}$ Y. Lei, L. Yu, B. Zhou, C. Zhu, Z. Wen, and S. H. Lin, "Landscapes of four-enantiomer conical intersections for photoisomerization of stilbene: CASSCF calculation," J. Phys. Chem. A 118, 9021 (2014).

${ }^{48}$ V. Molina, M. Merchán, and B. O. Roos, "Theoretical study of the electronic spectrum of trans-stilbene," J. Phys. Chem. A 101, 3478 (1997).

${ }^{49}$ A. E. Clark, "Time-dependent density functional theory studies of the photoswitching of the two-photon absorption spectra in stilbene, metacyclophenadiene, and diarylethene chromophores," J. Phys. Chem. A 110, 3790 (2006).

${ }^{50}$ V. Molina, M. Merchán, and B. O. Roos, "A theoretical study of the electronic spectrum of cis-stilbene," Spectrochim. Acta, Part A 55, 433 (1999).

${ }^{51} \mathrm{~B}$. Dick and G. Hohlneicher, "Two-photon excitation spectroscopy of phenanthrene singlet states below $50000 \mathrm{~cm}^{-1}$," Chem. Phys. Lett. 97, 324 (1983).

${ }^{52}$ A. Chakrabarti and S. I. Ramasesha, "Properties of the low-lying electronic states of phenanthrene: Exact PPP results," Int. J. Quantum Chem. 60, 381 (1996).

${ }^{53}$ R. González-Luque, L. Serrano-Andrés, M. Merchán, and M. P. Fülscher, "Theoretical characterization of the absorption spectra of phenanthrene and its radical cation," Theor. Chim. Acta 110, 224 (2003).

${ }^{54}$ R. J. Bartlett, "Many-body perturbation theory and coupled cluster theory for electron correlation in molecules," Annu. Rev. Phys. Chem. 32, 359 (1981)

${ }^{55}$ R. J. Bartlett and J. F. Stanton, “Applications of post-Hartree-Fock methods: A tutorial," Rev. Comput. Chem. 5, 65 (1994).

${ }^{56}$ R. J. Bartlett, "To multireference or not to multireference: That is the question?," Int. J. Mol. Sci. 3, 579 (2002).

${ }^{57}$ A. I. Krylov, "Equation-of-motion coupled-cluster methods for open-shell and electronically excited species: The Hitchhiker's guide to Fock space," Annu. Rev. Phys. Chem. 59, 433 (2008).

${ }^{58}$ R. J. Bartlett, "The coupled-cluster revolution," Mol. Phys. 108, 2905 (2010).

${ }^{59}$ K. Sneskov and O. Christiansen, "Excited state coupled cluster methods," Wiley Interdiscip. Rev.: Comput. Mol. Sci. 2, 566 (2012).

${ }^{60}$ R. J. Bartlett, "Coupled-cluster theory and its equation-of-motion extensions," Wiley Interdiscip. Rev.: Comput. Mol. Sci. 2, 126 (2012).

${ }^{61}$ F. Plasser and H. Lischka, "Analysis of excitonic and charge transfer interactions from quantum chemical calculations," J. Chem. Theory Comput. 8, 2777 (2012)

${ }^{62}$ F. Plasser, M. Wormit, and A. Dreuw, "New tools for the systematic analysis and visualization of electronic excitations. I. Formalism," J. Chem. Phys. 141, 024106 (2014).

${ }^{63}$ F. Plasser, S. A. Bäppler, M. Wormit, and A. Dreuw, "New tools for the systematic analysis and visualization of electronic excitations. II. Applications," J. Chem. Phys. 141, 024107 (2014).

${ }^{64}$ M. P. T. Beerepoot, D. H. Friese, N. H. List, J. Kongsted, and K. Ruud, "Benchmarking two-photon absorption cross sections: Performance of CC2 and CAM-B3LYP," Phys. Chem. Chem. Phys. 17, 19306 (2015).

${ }^{65} \mathrm{M}$. de Wergifosse, C. G. Elles, and A. I. Krylov, "Two-photon absorption spectroscopy of stilbene and phenanthrene: Excited-state analysis and comparison with ethylene and toluene," J. Chem. Phys. (submitted).

${ }^{66}$ A. L. Houk, I. L. Zheldakov, T. A. Tommey, and C. G. Elles, "Two-photon excitation of trans-stilbene: Spectroscopy and dynamics of electronically excited states above $S_{1}$, J. Phys. Chem. B 119, 9335 (2015). 
${ }^{67}$ C. G. Elles, C. A. Rivera, Y. Zhang, P. A. Pieniazek, and S. E. Bradforth, "Electronic structure of liquid water from polarization-dependent twophoton absorption spectroscopy," J. Chem. Phys. 130, 084501 (2009).

${ }^{68}$ R. A. Negres, J. M. Hales, A. Kobyakov, D. J. Hagan, and E. W. Van Stryland, "Two-photon spectroscopy and analysis with a white-light continuum probe," Opt. Lett. 27, 270 (2002).

${ }^{69}$ R. A. Negres, J. M. Hales, D. J. Hagan, and E. W. Van Stryland, "Experiment and analysis of two-photon absorption spectroscopy using a white-light continuum probe,” IEEE J. Quantum Electron. 38, 1205 (2002).

${ }^{70} \mathrm{~S}$. Yamaguchi and T. Tahara, "Two-photon absorption spectrum of all-trans retinal," Chem. Phys. Lett. 376, 237 (2003).

${ }^{71}$ C. Nagura, A. Suda, H. Kawano, M. Obara, and K. Midorikawa, "Generation and characterization of ultrafast white-light continuum in condensed media," Appl. Opt. 41, 3735 (2002).

${ }^{72}$ I. Buchvarov, A. Trifonov, and T. Fiebig, "Toward an understanding of white-light generation in cubic media-polarization properties across the entire spectral range," Opt. Lett. 32, 1539 (2007).

${ }^{73}$ A. L. Houk, "Nonlinear excitation of photoactivated molecules: Two-photon absorption spectroscopy, dynamics, and quantum yields," Ph.D. thesis, Department of Chemistry, University of Kansas, 2015.

${ }^{74} \mathrm{~T}$. Helgaker, S. Coriani, P. Jørgensen, K. Kristensen, J. Olsen, and K. Ruud, "Recent advances in wave function-based methods of molecular-property calculations," Chem. Rev. 112, 543 (2012).

${ }^{75}$ P. R. Monson and W. M. McClain, "Polarization dependence of the two photon absorption of tumbling molecules with application to liquid 1-chloronaphthalene and benzene," J. Chem. Phys. 53, 29 (1970).

${ }^{76}$ M. J. Wirth, A. Koskelo, and M. J. Sanders, "Molecular symmetry and 2-photon spectroscopy," Appl. Spectrosc. 35, 14 (1981).

${ }^{77}$ E. Epifanovsky, D. Zuev, X. Feng, K. Khistyaev, Y. Shao, and A. I. Krylov, "General implementation of resolition-of-identity and Cholesky representations of electron-repulsion integrals within coupled-cluster and equationof-motion methods: Theory and benchmarks," J. Chem. Phys. 139, 134105 (2013).

${ }^{78}$ A. I. Krylov and P. M. W. Gill, "Q-Chem: An engine for innovation," Wiley Interdiscip. Rev.: Comput. Mol. Sci. 3, 317 (2013).

${ }^{79}$ Y. Shao, Z. Gan, E. Epifanovsky, A. T. B. Gilbert, M. Wormit, J. Kussmann, A. W. Lange, A. Behn, J. Deng, X. Feng, D. Ghosh, M. Goldey, P. R. Horn, L. D. Jacobson, I. Kaliman, R. Z. Khaliullin, T. Kus, A. Landau, J. Liu, E. I. Proynov, Y. M. Rhee, R. M. Richard, M. A. Rohrdanz, R. P. Steele, E. J. Sundstrom, H. L. Woodcock III, P. M. Zimmerman, D. Zuev, B. Albrecht, E. Alguires, B. Austin, G. J. O. Beran, Y. A. Bernard, E. Berquist, K. Brandhorst, K. B. Bravaya, S. T. Brown, D. Casanova, C.M. Chang, Y. Chen, S. H. Chien, K. D. Closser, D. L. Crittenden, M. Diedenhofen, R. A. DiStasio, Jr., H. Do, A. D. Dutoi, R. G. Edgar, S. Fatehi, L. Fusti-Molnar, A. Ghysels, A. Golubeva-Zadorozhnaya, J. Gomes, M. W. D. Hanson-Heine, P. H. P. Harbach, A. W. Hauser, E. G. Hohenstein, Z. C. Holden, T.-C. Jagau, H. Ji, B. Kaduk, K. Khistyaev, J. Kim, J. Kim, R. A. King, P. Klunzinger, D. Kosenkov, T. Kowalczyk, C. M. Krauter, K. U. Laog, A. Laurent, K. V. Lawler, S. V. Levchenko, C. Y. Lin, F. Liu, E. Livshits, R. C. Lochan, A. Luenser, P. Manohar, S. F. Manzer, S.-P. Mao, N. Mardirossian, A. V. Marenich, S. A. Maurer, N. J. Mayhall, C. M. Oana, R. Olivares-Amaya, D. P. O’Neill, J. A. Parkhill, T. M. Perrine, R. Peverati, P. A. Pieniazek, A. Prociuk, D. R. Rehn, E. Rosta, N. J. Russ, N. Sergueev, S. M. Sharada, S. Sharmaa, D. W. Small, A. Sodt, T. Stein, D. Stuck, Y.-C. Su, A. J. W. Thom, T. Tsuchimochi, L. Vogt, O. Vydrov, T. Wang, M. A. Watson, J. Wenzel, A. White, C. F. Williams, V. Vanovschi, S. Yeganeh, S. R. Yost, Z.-Q. You, I. Y. Zhang, X. Zhang, Y. Zhou, B. R. Brooks, G. K. L. Chan, D. M. Chipman, C. J. Cramer, W. A. Goddard III, M. S. Gordon, W. J. Hehre, A. Klamt, H. F. Schaefer III, M. W. Schmidt, C. D. Sherrill, D. G. Truhlar, A. Warshel, X. Xu, A. Aspuru-Guzik, R. Baer, A. T. Bell, N. A. Besley,
J.-D. Chai, A. Dreuw, B. D. Dunietz, T. R. Furlani, S. R. Gwaltney, C.-P. Hsu, Y. Jung, J. Kong, D. S. Lambrecht, W. Z. Liang, C. Ochsenfeld, V. A. Rassolov, L. V. Slipchenko, J. E. Subotnik, T. Van Voorhis, J. M. Herbert, A. I. Krylov, P. M. W. Gill, and M. Head-Gordon, "Advances in molecular quantum chemistry contained in the Q-Chem 4 program package," Mol. Phys. 113, 184 (2015).

${ }^{80}$ R. S. Mulliken, "Report on notation for the spectra of polyatomic molecules," J. Chem. Phys. 23, 1997 (1955).

${ }^{81}$ Depending on molecular orientation, symmetry labels corresponding to the same orbital or vibrational mode may be different. Q-Chem's standard molecular orientation is different from that of Mulliken. ${ }^{80}$ For example, Q-Chem places the water molecule in the $x z$-plane instead of $y z$. Consequently, for $\mathrm{C}_{2} v$ symmetry, $b_{1}$ and $b_{2}$ labels are flipped. More details can be found at http://iopenshell.usc.edu/resources/howto/symmetry.

${ }^{82}$ A. L. Houk, R. S. Givens, and C. G. Elles, "Two-photon activation of p-hydroxyphenacyl phototriggers: Toward spatially controlled release of diethyl phosphate and ATP," J. Phys. Chem. B 120, 3178 (2016).

${ }^{83}$ W. M. McClain, "Excited state symmetry assignment through polarized two-photon absorption studies of fluids," J. Chem. Phys. 55, 2789 (1971).

${ }^{84}$ H. Larsen, K. Hald, J. Olsen, and P. Jørgensen, "Triplet excitation energies in full configuration interaction and coupled-cluster theory," J. Chem. Phys. 115, 3015 (2001).

${ }^{85}$ D. Zuev, K. B. Bravaya, T. D. Crawford, R. Lindh, and A. I. Krylov, "Electronic structure of the two isomers of the anionic form of p-coumaric acid chromophore," J. Chem. Phys. 134, 034310 (2011).

${ }^{86}$ E. Epifanovsky, K. Kowalski, P.-D. Fan, M. Valiev, S. Matsika, and A. I. Krylov, "On the electronically excited states of uracil," J. Phys. Chem. A 112, 9983 (2008).

${ }^{87}$ F. Vigliotti and M. Chergui, "Rydberg states in the condensed phase studied by fluorescence depletion spectroscopy," Eur. Phys. J. D 10, 379 (2000).

${ }^{88}$ J.-M. Mewes, Z.-Q. You, M. Wormit, T. Kriesche, J. M. Herbert, and A. Dreuw, "Experimental benchmark data and systematic evaluation of two a posteriori, polarizable-continuum corrections for vertical excitation energies in solution," J. Phys. Chem. A 119, 5446 (2015).

${ }^{89}$ Z. Q. You, J. M. Mewes, A. Dreuw, and J. M. Herbert, "Comparison of the Marcus and Pekar partitions in the context of non-equilibrium, polarizablecontinuum solvation models,” J. Chem. Phys. 143, 204104 (2015).

${ }^{90}$ K. B. Bravaya and A. I. Krylov, "On the photodetachment from the green fluorescent protein chromophore," J. Phys. Chem. A 117, 11815 (2013).

${ }^{91} \mathrm{H}$. Reisler and A. I. Krylov, "Interacting Rydberg and valence states in radicals and molecules: Experimental and theoretical studies," Int. Rev. Phys. Chem. 28, 267 (2009).

${ }^{92}$ E. Kamarchik and A. I. Krylov, "Non-Condon effects in one- and twophoton absorption spectra of the green fluorescent protein,” J. Phys. Chem. Lett. 2, 488 (2011).

${ }^{93}$ N. Lin, Y. Luo, K. Ruud, X. Zhao, F. Santoro, and A. Rizzo, "Differences in two-photon and one-photon absorption profiles induced by vibronic coupling: The case of dioxaborine heterocyclic dye," Comput. Phys. Commun. 12, 3392 (2011).

${ }^{94}$ M. S. Gordon, Q. A. Smith, P. Xu, and L. V. Slipchenko, "Accurate first principles model potentials for intermolecular interactions," Annu. Rev. Phys. Chem. 64, 553-578 (2013).

${ }^{95}$ D. Ghosh, D. Kosenkov, V. Vanovschi, C. Williams, J. Herbert, M. S. Gordon, M. Schmidt, L. V. Slipchenko, and A. I. Krylov, "Non-covalent interactions in extended systems described by the effective fragment potential method: Theory and application to nucleobase oligomers," J. Phys. Chem. A 114, 12739 (2010).

${ }^{96}$ L. V. Slipchenko, "Solvation of the excited states of chromophores in polarizable environment: Orbital relaxation versus polarization,” J. Phys. Chem. A 114, 8824 (2010). 